\title{
MULTILEVEL NEURAL GRADIENTS REFLECT TRANSDIAGNOSTIC EFFECTS OF MAJOR PSYCHIATRIC CONDITIONS ON CORTICAL MORPHOLOGY
}

Bo-yong Park ${ }^{1,2,3^{*}}$, Valeria Kebets ${ }^{1}$, Sara Larivière ${ }^{1}$, Meike D. Hettwer ${ }^{4,5,6}$, Casey Paquola ${ }^{1,7}$, Daan van Rooij ${ }^{8,9}$, Jan Buitelaar ${ }^{10}$, Barbara Franke ${ }^{10,11}$, Martine Hoogman ${ }^{10,11}$, Lianne Schmaal ${ }^{12,13}$, Dick J. Veltman ${ }^{14}$, Odile van den Heuvel ${ }^{14}$, Dan J. Stein ${ }^{15}$, Ole A. Andreassen ${ }^{16,17}$, Christopher R. K. Ching ${ }^{18}$, Jessica Turner ${ }^{19,20,21}$, Theo G. M. van $\operatorname{Erp}^{22,23}$, Alan C. Evans ${ }^{1}$, Alain Dagher ${ }^{1}$, Sophia I. Thomopoulos $^{24}$, Paul M. Thompson ${ }^{24}$, Sofie L. Valk ${ }^{4,5}$, Matthias Kirschner ${ }^{1,25 \#}$, Boris C. Bernhardt ${ }^{1 * \#}$

${ }^{1}$ McConnell Brain Imaging Centre, Montreal Neurological Institute and Hospital, McGill University, Montreal, Quebec, Canada

${ }^{2}$ Department of Data Science, Inha University, Incheon, Republic of Korea

${ }^{3}$ Center for Neuroscience Imaging Research, Institute for Basic Science, Suwon, Republic of Korea

${ }^{4}$ Forschungszentrum Julich, Germany

${ }^{5}$ Max Planck Institute for Cognitive and Brain Sciences, Leipzig, Germany

${ }^{6}$ Max Planck School of Cognition, Leipzig, Germany

${ }^{7}$ INM-1, Forschungszentrum Julich, Germany

${ }^{8}$ Center for Cognitive Neuroimaging, Donders Institute for Brain, Cognition and Behavior, Radboud University, Nijmegen, The Netherlands

${ }^{9}$ Department of Psychiatry, University of Groningen, University Medical Center Groningen, Groningen, The Netherlands

${ }^{10}$ Donders Institute for Brain, Cognition and Behaviour, Radboud University, Nijmegen, The Netherlands

${ }^{11}$ Department of Human Genetics \& Psychiatry, Radboud university medical center, Nijmegen, The Netherlands

${ }^{12}$ Centre for Youth Mental Health, The University of Melbourne, Melbourne, Australia

${ }^{13}$ Orygen, Parkville, Australia

${ }^{14}$ Departments of Psychiatry and Anatomy and Neuroscience, Amsterdam University Medical Centers, Vrije Universiteit Amsterdam, Amsterdam, The Netherlands

${ }^{15}$ SA Medical Research Council Unit on Risk \& Resilience in Mental Disorders, Dept of Psychiatry and Mental Health, University of Cape Town, Cape Town, South Africa

${ }^{16}$ NORMENT Centre, Division of Mental Health and Addiction, Oslo University Hospital \&Institute of Clinical Medicine, University of Oslo, Oslo, Norway

${ }^{17} \mathrm{KG}$ Jebsen Centre for Neurodevelopment, Institute of Clinical Medicine, University of Oslo, Oslo, Norway

${ }^{18}$ Imaging Genetics Center, Mark and Mary Stevens Neuroimaging and Informatics Institute, Keck School of Medicine, University of Southern California, USA

${ }^{19}$ Department of Psychology, Georgia State University, USA

${ }^{20}$ Tri-institutional Center for Translational Research in Neuroimaging and Data Science (TReNDS), Georgia State University, Georgia Institute of Technology and Emory University, USA

${ }^{21}$ Neuroscience Institute, Georgia State University, USA

${ }^{22}$ Clinical Translational Neuroscience Laboratory, Department of Psychiatry and Human Behavior, University of California Irvine, Irvine, California, USA

${ }^{23}$ Center for the Neurobiology of Learning and Memory, University of California Irvine, Irvine, California, USA

${ }^{24}$ Imaging Genetics Center, Mark and Mary Stevens Neuroimaging and Informatics Institute, Keck School of Medicine,

University of Southern California, Los Angeles, California, United States of America

${ }^{25}$ Department of Psychiatry, Psychotherapy and Psychosomatics, Psychiatric Hospital, University of Zurich,

Switzerland;

\# These authors contributed equally to the work.

\section{* Corresponding Authors:}

Bo-yong Park, PhD

Department of Data Science

Inha University

Incheon, Republic of Korea

Phone: +82-32-860-9427

Email: boyong.park@inha.ac.kr
Boris C. Bernhardt, $\mathrm{PhD}$

Multimodal Imaging and Connectome Analysis Lab

McConnell Brain Imaging Centre

Montreal Neurological Institute and Hospital

McGill University

Montreal, Quebec, Canada

Phone: +1-514-398-3579

Email: boris.bernhardt@mcgill.ca 
Park et al. | Cross-condition effects of major psychiatric conditions

\begin{abstract}
It is increasingly recognized that multiple psychiatric conditions are underpinned by shared neural pathways, affecting similar brain systems. Here, we assessed $i$ ) shared dimensions of alterations in cortical morphology across six major psychiatric conditions (autism spectrum disorder, attention deficit/hyperactivity disorder, major depression, obsessive-compulsive disorder, bipolar disorder, schizophrenia) and $\mathrm{ii}$ ) carried out a multiscale neural contextualization, by cross-referencing shared anomalies against cortical myeloarchitecture and cytoarchitecture, as well as connectome and neurotransmitter organization. Pooling disease-related effects on MRI-based cortical thickness measures across six ENIGMA working groups, including a total of 28,546 participants $(12,876$ patients and 15,670 controls), we computed a shared disease dimension on cortical morphology using principal component analysis that described a sensory-fugal pattern with paralimbic regions showing the most consistent abnormalities across conditions. The shared disease dimension was closely related to cortical gradients of microstructure and intrinsic connectivity, as well as neurotransmitter systems, specifically serotonin and dopamine. Our findings embed the shared effects of major psychiatric conditions on brain structure in multiple scales of brain organization and may provide novel insights into neural mechanisms into transdiagnostic vulnerability.
\end{abstract}

KEYWORDS: psychiatric condition; cortical thickness; gradient; cytoarchitecture; neurotransmitter; multisite; multiscale 
bioRxiv preprint doi: https://doi.org/10.1101/2021.10.29.466434; this version posted November 2,2021 . The copyright holder for this preprint (which was not certified by peer review) is the author/funder, who has granted bioRxiv a license to display the preprint in perpetuity. It is made available under aCC-BY-NC-ND 4.0 International license.

Park et al. | Cross-condition effects of major psychiatric conditions

\section{INTRODUCTION}

Mental illness refers to a wide range of psychiatric conditions, affecting individuals, families, and health systems at large [1]. While conventional psychiatric nosology classifies mental illness into distinct categories mainly based on descriptive symptoms and behaviors [2], high co-occurrence of symptoms across disorders as well as transdiagnostic risk factors have prompted reconceptualization of mental illnesses along symptom dimensions [3-8]. The dimensional framework benefits detailed characterization of individual variations, and may allow for more direct brain-behavior associations than classic case-control comparisons that capture multiple symptom classes and mask clinical heterogeneity.

The shared components across major psychiatric diagnosis may be more clearly distinguishable at the neural level $[4,9]$, as the behavioral level likely involves complex interactions with society and the environment [10]. Structural magnetic resonance imaging (MRI), in particular, offers high spatial precision to help resolve the pattern of shared transdiagnostic effects across the cortical surface [4, 11-16]. A large body of prior case-control studies has reported reproducible patterns of structural alterations in cohorts with psychiatric diagnoses relative to controls [17-21], often pointing to widespread changes in cortical morphology in these conditions. More recently, efforts have been expanded to a transdiagnostic perspective, aiming to identify structural compromise that are shared across different diagnoses [22-24]. To ensure sensitivity of such efforts and to strengthen reproducibility, it becomes increasingly relevant to pool these investigations across multiple sites. One such initiative, spearheaded by the Enhancing NeuroImaging Genetics through Meta-Analysis (ENIGMA) consortium, has aggregated MRI and phenotypic data in thousands of healthy individuals and those with a psychiatric diagnosis [25]. Moreover, dedicated ENIGMA working groups have confirmed neuroanatomical disruptions in major psychiatric indications, including autism spectrum disorder (ASD) [26], attention deficit hyperactivity disorder (ADHD) [27], major depressive disorder (MDD) [28], obsessive-compulsive disorder (OCD) [29], bipolar disorder (BD) [30], and schizophrenia (SZ) [31], pointing to widespread changes in cortical morphology in each of these different conditions.

In addition to providing robust evidence of neuroanatomical signatures associated with each of these conditions, an emerging body of studies has pooled data across different indications to identify shared anomalies of psychiatric conditions [32, 33]. In an effort to identify factors contributing to the topography of cross-disorder brain changes, a recent study has taken this approach one step further and examined associations to post mortem gene expression data, searching for spatially co-varying gene lists that may carry susceptibility to transdiagnostic disease effects. This study identified that transdiagnostic effects may specifically be present in regions with greater expression of CA1 pyramidal genes that were suggested to play a role in regulating cortical thickness. Beyond these molecular risk factors, there is a broad range of cellular, metabolic, and functional properties of brain regions that may contribute to the regional susceptibility of transdiagnostic disease effects. An influential theory, also referred to as the structural model, posits that the internal microstructural and connectional markup of different brain regions, in particular their laminar differentiation and corticocortical connectivity patterns, may represent mesoscale features associated with the potential of a region to show plasticity, and to be susceptible to pathological processes [34]. According to this framework, paralimbic cortices with low laminar differentiation and higher-order connectivity profiles may be more susceptible to effects of neurological as well as psychiatric disorders. Here, we tested this approach, by aligning transdiagnostic effects with maps of microstructural variations derived from both in vivo imaging and 3D post mortem histology [35-38]. In recent work, the application of non-linear eigenvector decomposition to these datasets identified a "sensory-fugal" gradient that radiates from sensory and motor areas with strong laminar differentiation and higher myelination towards heteromodal association and paralimbic regions with less clear lamination and 
Park et al. | Cross-condition effects of major psychiatric conditions

lower myelin content. Of note, similar gradients have also been derived from the analysis of intrinsic functional connectivity patterns obtained from resting-state functional MRI [37-39]. In line with foundational neuroanatomical conceptualization [34, 40, 41], an emerging literature has underscored a correspondence between such data-driven sensory-fugal gradients, and region-to-region variations in cortical plasticity and genetic control [39, 42-46], suggesting that these likely help understand susceptibility to disease as well [39, 42, 47-51].

The study of micro- and macroscale cortical organization as well as the identification of factors contributing to disease-related susceptibility for psychiatric conditions can be further complemented by studying associations to the neurotransmitter architecture of the human brain. Recent work based on in vitro receptor autoradiography in non-human primates has suggested that neurotransmitter systems are likely organized along similar gradients as cortical microstructure and connectivity, enabling on the one hand rapid and reliable information processing in sensory areas on the one hand, and slow, flexible integration of information in higher cognitive areas. Until similar resources become available in humans, one can approximate the spatial distributions of different neurotransmitter systems in vivo, based on the aggregation of positron emission tomography (PET) and single photon computed emission tomography (SPECT) studies sensitive to different receptor an transporter types [52-58]. Such mapping can thus provide a molecular perspective to complement microstructural and functional connectivity contextualization of transdiagnostic findings, promising new insights into factors contributing to the susceptibility of the brain to effects of different psychiatric conditions.

Here, we studied the association between multiscale neural organization and transdiagnostic effects on cortical morphology across six major psychiatric conditions, which represent a broad range of common and severe neurodevelopmental indications (ASD, ADHD, MDD, OCD, BD, and SZ). Aggregating data from thousands of patients and healthy controls previously studied across several ENIGMA working groups [26-31], we defined shared effects using principal component analysis, adapting a previous framework [32], and then associated the effects across multiple neural scales, namely (i) in vivo myeloarchitecture and intrinsic functional connectivity, (ii) post mortem 3D cytoarchitecture, and (iii) in vivo maps of neurotransmitter distributions.

\section{RESULTS}

\section{Study overview and participants}

We obtained case-control maps of cortical thickness differences in patients relative to controls, resulting from several ENIGMA meta-analyses provided by a previous study, aggregating a total of 28,546 participants across 145 independent cohorts (1,821 ASD, 1,815 ADHD, 2,695 MDD, 2,274 OCD, 1,555 BD, 2,716 SZ; 15,670 site-matched controls Table S1) [32]. We then associated principal dimensions of morphological abnormalities with (i) in vivo myeloarchitecture and functional connectivity gradients obtained from the Human Connectome Project (HCP) [59], (ii) post mortem cytoarchitecture, by cross-referencing data to a ultra-high resolution 3D histological reconstruction of a human brain [60], and (iii) in vivo neurotransmitter topographies provided by PET/SPECT studies [52-58]. Approaches are openly available and replicable via the ENIGMA toolbox (https://enigma-toolbox.readthedocs.io) [61]. See Methods for more details.

\section{Shared dimensions of structural alterations across psychiatric conditions}

Following standardized ENIGMA protocols (http://enigma.ini.usc.edu/protocols/imaging-protocols/), gray matter thickness for 68 cortical regions of the Desikan-Killany atlas [62] was calculated, and meta-analytic between-group differences in cortical thickness were assessed using inverse varianceweighted random-effects models (Fig. 1A) [32]. Using principal component analysis adopted in a 
bioRxiv preprint doi: https://doi.org/10.1101/2021.10.29.466434; this version posted November 2, 2021. The copyright holder for this preprint (which was not certified by peer review) is the author/funder, who has granted bioRxiv a license to display the preprint in perpetuity. It is made available under aCC-BY-NC-ND 4.0 International license.

Park et al. | Cross-condition effects of major psychiatric conditions

recent study [32], we then estimated the shared disease dimensions explaining structural alterations across six conditions (Fig. 1B). The first dimension/component explained $55.7 \%$ of variance, and differentiated sensory/motor systems having positive scores from transmodal/paralimbic areas with negative scores (for details, and information on the other dimensions/components, see Fig. S1A). Stratifying the first dimension according to intrinsic functional communities [63], it indeed differentiated somatomotor/visual from default/frontoparietal/limbic networks (Fig. 1B). Similar spatial patterns were observed across the levels of the putative primate cortical hierarchy [40], differentiating idiotypic/unimodal from heteromodal/paralimbic levels. Notably, scores on the principal dimension translated into mean effect sizes across case-control analyses, with paralimbic regions showing strongest atrophy in patients relative to controls, while sensory/motor regions showed the least gray matter alterations (Fig. S1B). We also directly ran principal component analysis on previously reported effect size maps (Cohen's d) concatenated across disorders, sourced from the ENIGMA toolbox [61] (Fig. S1C). Findings were highly similar, suggesting robustness. The shared disease effect resembled the effects of each condition, with the strongest spatial similarity to SZ and $\mathrm{BD}$, followed by MDD, ADHD, ASD, and OCD (spin-test followed by false discovery rate (FDR) correction, $p_{\text {spin-FDR }}<0.001$; Fig. S2), indicating that the shared effect captured structural alterations from each condition. We furthermore re-evaluated the shared dimension using leave-one-conditionout procedure (see Methods), and observed largely consistent results with the shared effect based on all conditions $\left(r>0.9 p_{\text {spin-FDR }}<0.001\right.$; Fig. S3), indicating that a single condition with strong metaanalytic profile did not determine the shared disease effect. 
bioRxiv preprint doi: https://doi.org/10.1101/2021.10.29.466434; this version posted November 2, 2021. The copyright holder for this preprint (which was not certified by peer review) is the author/funder, who has granted bioRxiv a license to display the preprint in perpetuity. It is made available under aCC-BY-NC-ND 4.0 International license.

Park et al. | Cross-condition effects of major psychiatric conditions

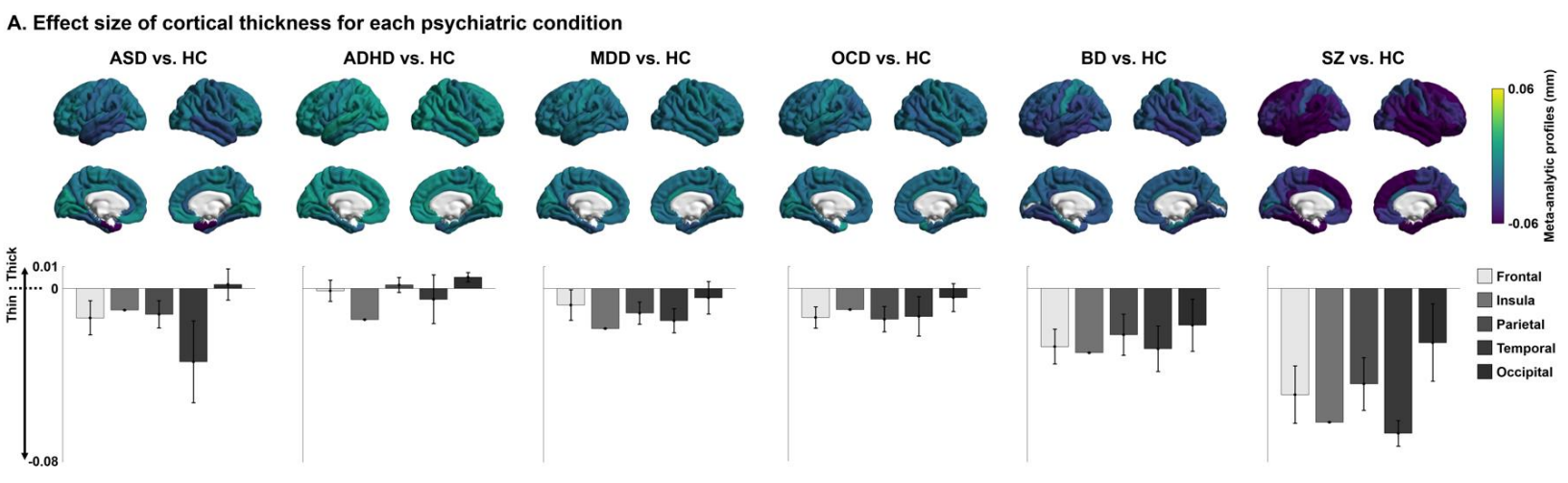

B. Shared effect


C. Gradients

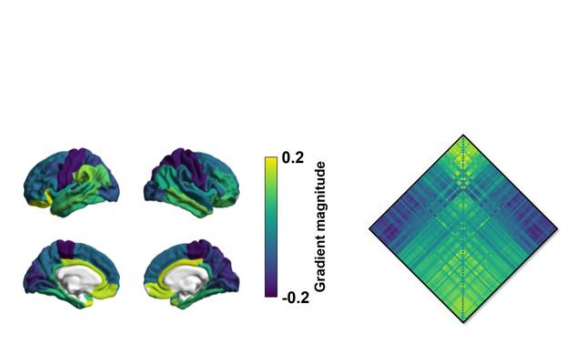

D. Association with shared effect
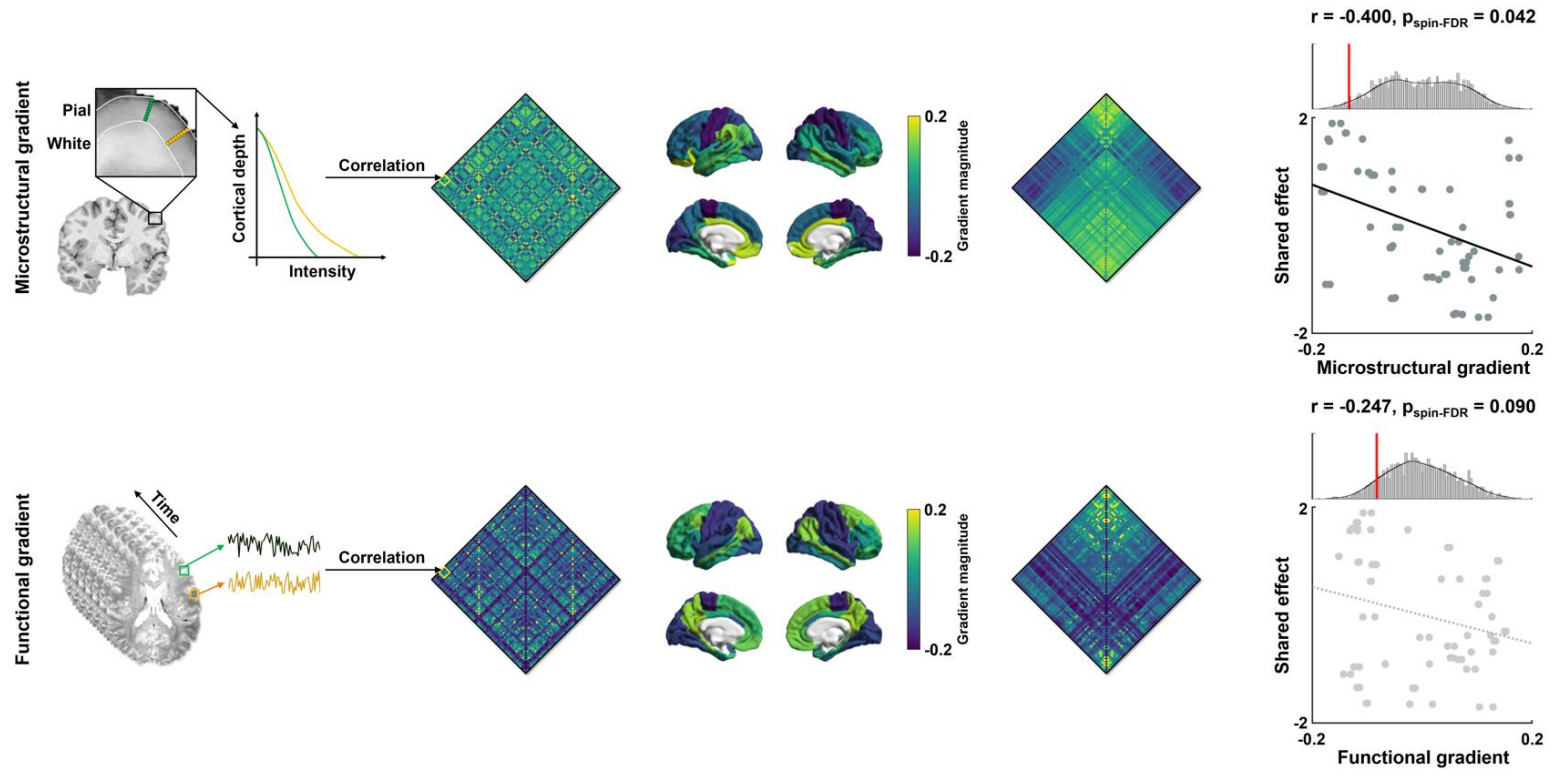

Fig. 1 | Shared disease effect and associations to connectivity gradients. (A) Meta-analytic profiles of cortical thickness differences (unit in $\mathrm{mm}$ ) in patients with each psychiatric condition relative to matched controls. Positive/negative values indicate increases/decreases in cortical thickness in patients relative to controls. Mean values of the regions involved in the same cortical lobes with SD are reported with bar plots. (B) The shared effect was identified through principal component analysis (PCA) applied to the concatenated effect size map. Spider plots stratify the effects according to functional communities [63] and cortical hierarchy levels [40]. (C) The microstructural and functional connectivity gradients were generated by applying non-linear dimensionality reduction techniques to the group averaged connectivity matrix (middle left), and each connectivity matrix was reordered (right) according to the first gradients (middle right). (D) Spatial correlation of each gradient with the shared effect map are shown in the scatter plots. The distribution of correlation coefficients across 1,000 spin-tests are reported with histograms, and the actual $\mathrm{r}$-values are represented with red bars. Abbreviations: ASD, autism spectrum disorder; ADHD, attention deficit hyperactivity disorder; MDD, major depressive disorder; OCD, obsessive-compulsive disorder; BD, bipolar disorder; SZ, schizophrenia; HC, healthy controls. 
Park et al. | Cross-condition effects of major psychiatric conditions

\section{Associations with cortical myeloarchitecture and functional connectivity gradients}

To assess in vivo micro- and macroscopic properties of the shared disease dimension on cortical morphology, we first examined its association with myeloarchitecture and intrinsic functional connectivity gradients [37, 39] (see Methods; Fig. 1C). The microstructural gradient was derived from inter-regional similarity matrices of intracortical profiles of myelin-sensitive MRI [37] , and runs from sensory/motor regions with high laminar differentiation and high intracortical myelin content towards paralimbic cortices with reduced laminar differentiation and low myelin content [37]. The intrinsic functional gradient was derived from resting-state functional MRI connectivity. While it also runs from sensory to transmodal areas, it finds its apex in the heteromodal default mode and frontoparietal networks, and not in paralimbic cortices [39]. Associating the patterns of shared dimension with these two in vivo gradients, we observed a negative association with the microstructural gradient $\left(\mathrm{r}=-0.400, \mathrm{p}_{\text {spin-FDR }}=0.042\right)$ and a negative trend with the functional connectivity gradient $\left(r=-0.247, p_{\text {spin-FDR }}=0.090\right.$; Fig. 1C). In other words, transdiagnostic morphological alterations follow sensory-fugal gradients of cortical organization, in particular the microstructural gradient that differentiates sensory/motor areas with high myelination and distinct lamination from paralimbic areas with low myelin content and reduced laminar differentiation.

\section{Cytoarchitectonic associations}

We furthermore examined associations with cortical cytoarchitecture [36], using a 3D histological reconstruction of a post mortem human brain, the BigBrain [60, 64]. We calculated cortex-wide variations in cytoarchitecture using two alternative approaches. First, we obtained intracortical intensity profiles and calculated their statistical moments, i.e., mean, SD, skewness, and kurtosis (Fig. 2A-B). In both classic cytoarchitecture analysis and more recent work, these features have been shown to relate to inter-areal microstructural differentiation $[38,65]$. For example, the skewness moment describes spatial transition from areas with low laminar differentiation and negative skewness to those with high laminar differentiation and positive skewness [65-67]. Moreover, we computed externopyramidization [68], describing gradual shift of intensity profiles across cortical layers that has been suggested to differentiate areas on the lower end of the cortical hierarchy from those that are higher up due to hierarchical shifts in laminar projection profiles [69] (Fig. 2A-B). Spatial correlations between these features and the principal disease dimension indicated relations to both profile skewness $\left(r=0.400, p_{\text {spin-FDR }}=0.015\right)$ and externopyramidization $\left(r=0.472, \mathrm{p}_{\text {spin-FDR }}=\right.$ 0.015; Fig. 2C). In other words, transdiagnostic alteration in cortical morphology was more likely in paralimbic regions with low skewness and low externopyramidization, independently confirming that those areas with low laminar differentiation were more likely to show transdiagnostic cortical alterations. 
Park et al. | Cross-condition effects of major psychiatric conditions

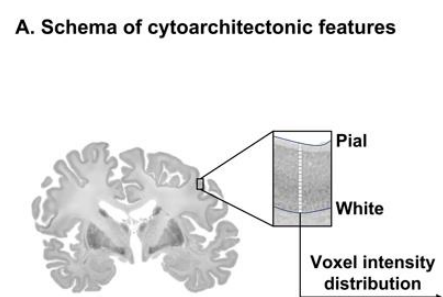

B. Cytoarchitectonic features Mean

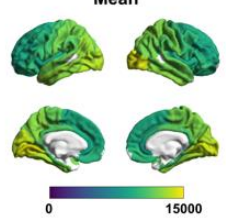

C. Association with shared effect

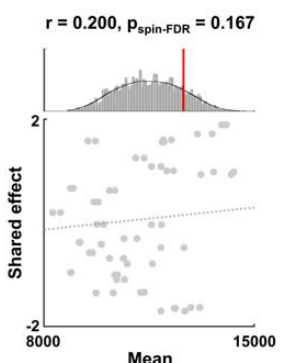

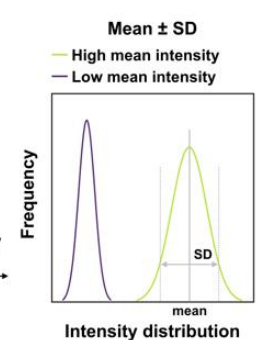

SD
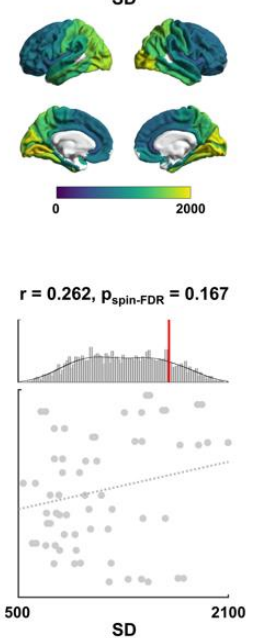

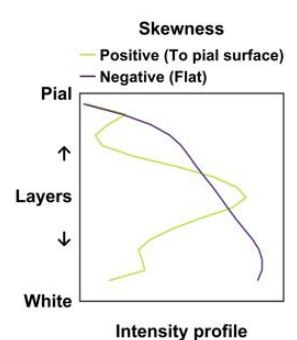

Intensity profile
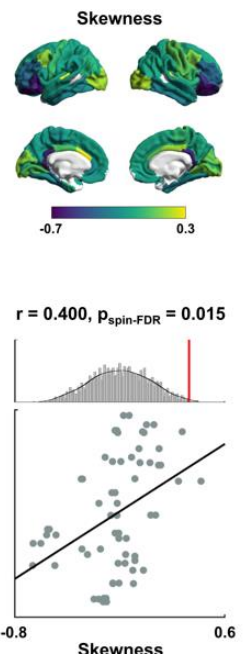

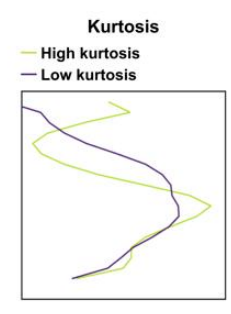

Intensity profile
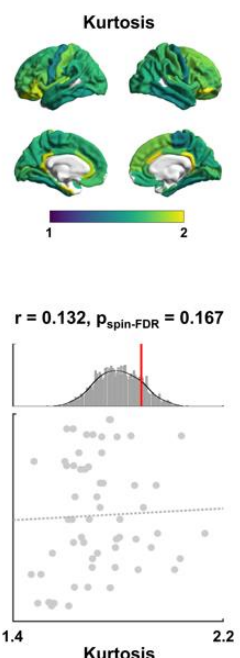
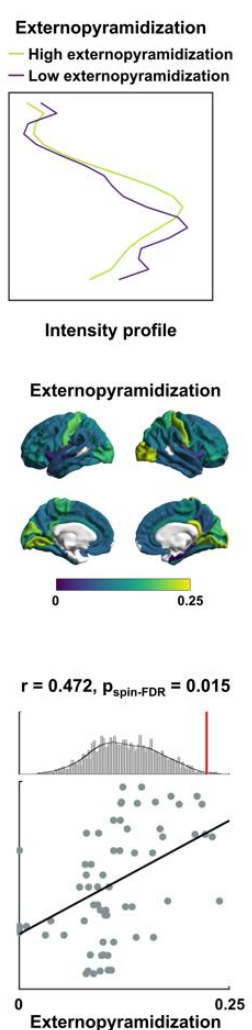

Fig. 2 Cytoarchitectonic associations with the shared disease effect. (A) Cytoarchitectonic moment features of mean, $\mathrm{SD}$, skewness, and kurtosis, as well as externopyramidization of intracortical intensity profile were calculated from the post mortem human brain, and (B) plotted on brain surfaces. (C) Spatial correlations between the features and shared effects are shown on scatter plots. The distributions of correlation coefficients across 1,000 spin-tests are reported with histograms, and the actual r-values are represented with red bars. Abbreviation: SD, standard deviation.

\section{Associations with distributions of neurotransmitter systems}

Neurotransmitter contextualization leveraged JuSpace [52], a toolbox that disseminates in vivo PET/SPECT data sensitive to ten different transmitters/transporters/receptors from independent studies in healthy human adults [53-58] (Fig. 3A). Associating the shared dimension with cortexwide neurotransmitter maps, we observed positive associations with D2 and 5-HT1b receptor densities (D2: $\left.\mathrm{r}=0.280, \mathrm{p}_{\text {spin-FDR }}=0.035 ; 5-\mathrm{HT} 1 \mathrm{~b}: \mathrm{r}=0.349, \mathrm{p}_{\text {spin-FDR }}=0.025\right)$, and negative correlations with dopamine transporter and 5-HT1a receptor density $\left(\mathrm{DAT}: \mathrm{r}=-0.240, \mathrm{p}_{\text {spin-FDR }}=\right.$ 0.041 ; 5-HT1a: $\mathrm{r}=-0.307, \mathrm{p}_{\text {spin-FDR }}=0.033$; Fig. 3B). The results indicate that common cortical abnormality patterns across psychiatric and neurodevelopmental conditions may be reflected by serotonergic and dopaminergic systems. More specifically, higher transdiagnostic cortical atrophy was related to higher 5-HT1a and lower 5-HT1b, as well as higher DAT and lower D2 receptor density. 
bioRxiv preprint doi: https://doi.org/10.1101/2021.10.29.466434; this version posted November 2, 2021. The copyright holder for this preprint (which was not certified by peer review) is the author/funder, who has granted bioRxiv a license to display the preprint in perpetuity. It is made available under aCC-BY-NC-ND 4.0 International license.

Park et al. | Cross-condition effects of major psychiatric conditions

\section{A. Schema of neurotransmitter systems}

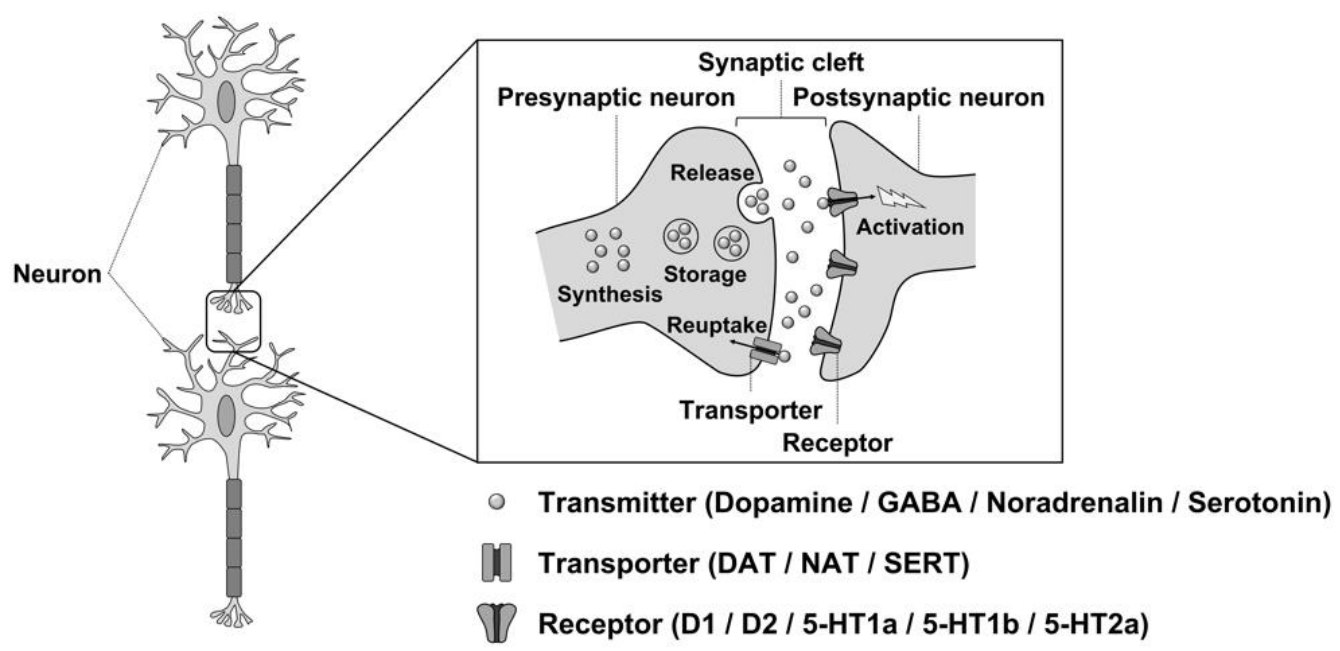

B. Association with shared effect

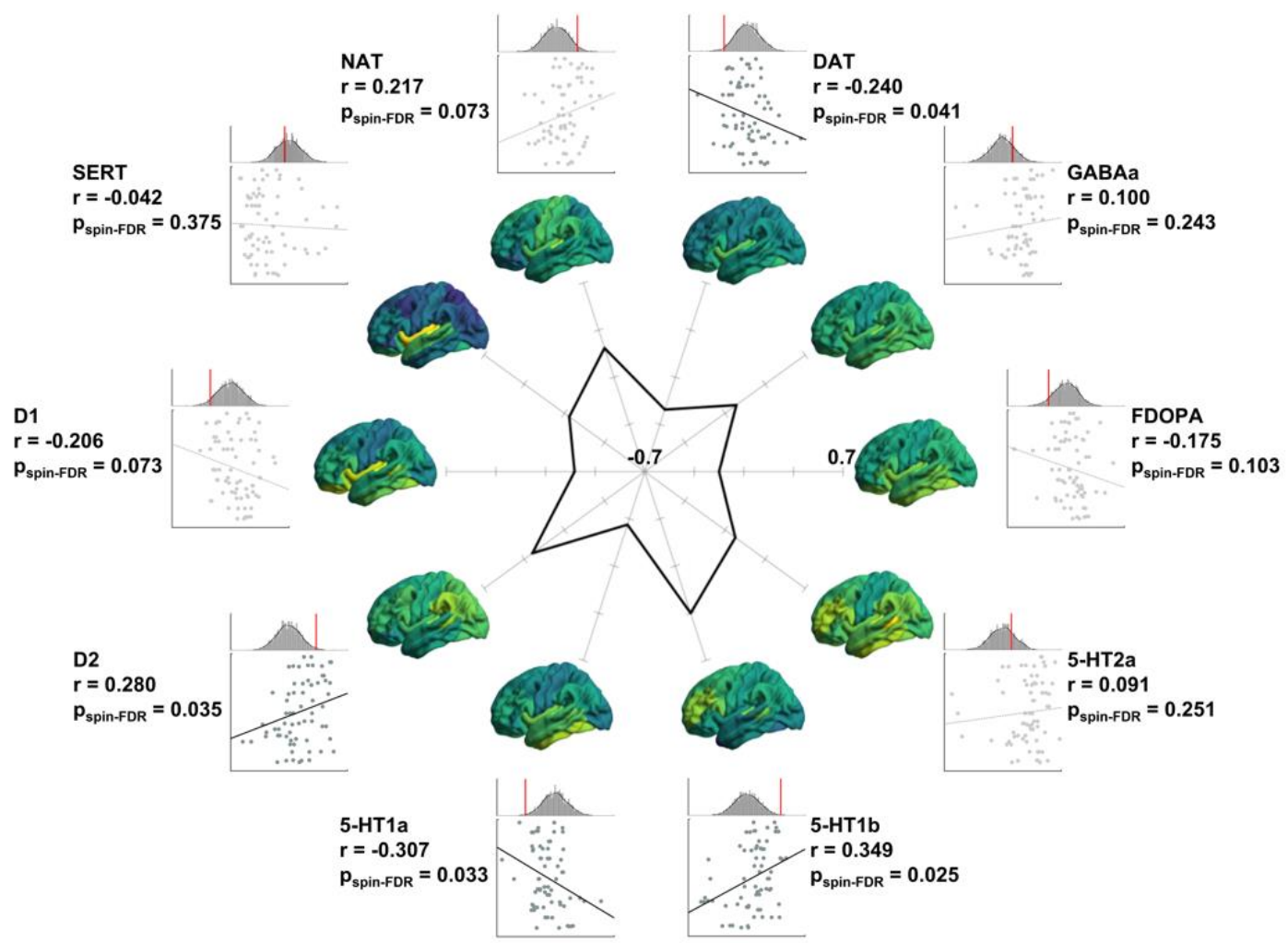

Fig. 3 Associations of neurotransmitter systems with shared disease effect. (A) Schema of neurotransmitter systems of transmitters, transporters, and receptors. (B) Spatial correlations of each neurotransmitter map with shared effect are shown on scatter plots. The distributions of correlation coefficients across 1,000 spin-tests are reported with histograms, and actual r-values are reported with red bars. The spider plot shows correlation coefficients. Cortex-wide spatial maps of the transmitter systems are reported on brain surfaces. Abbreviations: FDOPA, 18F fluorodopa; DAT, dopamine transporter; NAT, noradrenaline transporter; SERT, serotonin transporter.

\section{Prediction of the shared disease effect}

As a final analysis, we used supervised machine learning to predict the shared dimension using the above multiscale features. Specifically, we leveraged least absolute shrinkage and selection operator (LASSO) regression [70] with five-fold nested cross-validation [71-74] to predict the cross-condition effect using concatenated multiscale features (see Methods; Fig. 4A). Repeating the analysis for 100 
Park et al. | Cross-condition effects of major psychiatric conditions

times with different training and test dataset subsplits, we could reliably predict the spatial pattern of the shared disease dimension (mean $\pm \mathrm{SD} \mathrm{r}=0.518 \pm 0.044$, mean absolute error $(\mathrm{MAE})=0.828 \pm$ 0.039 , pperm $<0.001$; Fig. 4B). Cytoarchitectural skewness and externopyramidization, followed by D2 and 5-HT1b receptors, as well as the microstructural gradient were frequently selected across cross-validations and repetitions (Fig. 4A). When considering each psychiatric condition separately, we could find significant prediction performances, but the features selected diverge across conditions (Fig. S4).

A. Selected probability

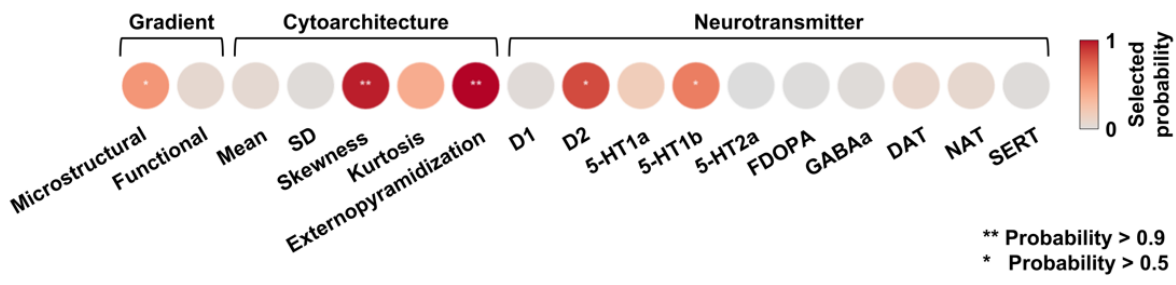

B. Prediction of shared effect

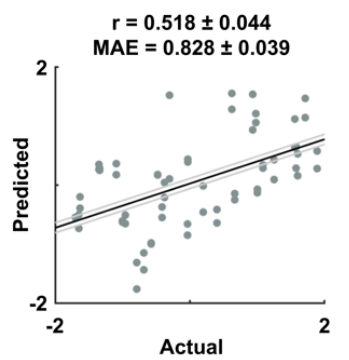

Fig. 4 Association between the shared disease effect and multiscale features using machine learning. (A) Probability of the selected features across five-fold nested cross-validations and 100 repetitions for predicting the shared disease effect. The frequently selected features are reported with asterisks. (B) Linear correlation between actual and predicted values of the effects is shown on a scatter plot. Black line indicates mean correlation and gray lines represent the $95 \%$ confidence interval for 100 iterations with different training/test datasets. Abbreviations: SD, standard deviation; FDOPA, 18F fluorodopa; DAT, dopamine transporter; NAT, noradrenaline transporter; SERT, serotonin transporter; MAE, mean absolute error.

\section{DISCUSSION}

The current work determined cortex-wide variations in susceptibility to morphological alterations across six major psychiatric conditions (i.e., ASD, ADHD, MDD, OCD, BD, and SZ), and crossreferenced these spatial patterns against multiscale cortical organization. Specifically, studying data aggregated by several multi-site ENIGMA consortia on the above indications [26-32], we identified a shared morphological dimension that followed a sensory-fugal pattern of increasing susceptibility to morphological alterations in paralimbic regions. Moreover, we cross-referenced these findings against neural axes previously described by (i) in vivo MRI measures sensitive to cortical myeloarchitecture and intrinsic functional connectivity [37, 39], (ii) post mortem histological measures sensitive to cytoarchitecture, in particular laminar differentiation [36, 38, 60, 65], and (iii) in vivo PET/SPECT derived measures of cortical neurotransmitter systems [52-58]. Our findings revealed that the transdiagnostic dimension of morphological anomalies closely aligned with microstructural gradients differentiating sensory/motor from paralimbic areas on the basis of cortical cyto- and myeloarchitecture, together as well as the variable distribution of serotonin and dopamine neurotransmitter systems. By offering new insights into multiscale neural features that align with cortical structural compromise across several psychiatric conditions, our work outlines micro- and macroscale determinants of cerebral vulnerability to the effects of common mental illnesses.

Complementing earlier case-control MRI studies performed separately in common neuropsychiatric conditions [17, 26-31, 75], an emerging literature of both primary observational studies [22-24] as well as meta-analyses [32, 33] has increasingly investigated sets of disorders to explore transdiagnostic effects on brain structure. Recently, these studies were complemented by dimensional data decomposition approaches of cortical morphological data, for example a recent factor analysis 
Park et al. | Cross-condition effects of major psychiatric conditions

[33] and principal component analyses [32]. We extended these prior studies by calculating mean effect size of the previously published condition-specific effects [32], as well as applying principal component analysis to the effect sizes and those sourced from the ENIGMA toolbox [61], confirming robust patterns. Specifically, the first shared dimension of cortical morphological alterations described a gradual axis running from sensory-motor regions at one end towards transmodal and most specifically paralimbic areas on the other end. In a prior study, the shared dimension was crossreferenced against gene expression information from the Allen Brain Institute [76-78], a comprehensive microarray-derived transcriptomics dataset based on four post mortem brains in the left and two in both left and right hemispheres. Using this resource, the authors found that transdiagnostic effects were highest in brain regions expressing genes for pyramidal cornu Ammonis 1 (CA1) cells, a finding that may already point towards a potentially increased susceptibility of limbic allocortices to transdiagnostic effects on brain morphology [32]. Here, we extended these findings by contextualizing the shared disease effect across multiple scales of neural organization, including cortex-wide variations in myeloarchitecture, cytoarchitecture, intrinsic functional connectivity, as well as neurotransmitter distributions.

The in vivo microstructural cortical gradient was defined using a recently-introduced procedure [37], which identified axes of cortico-cortical differentiation based on the similarity of myelin-sensitive MRI profiles sampled across cortical depths. In healthy adults and adolescents [37, 65], this approach has revealed a robust sensory-fugal cortical gradient running from sensory/motor areas with marked laminar differentiation and high myelin content towards paralimbic cortices with low overall myelination and rather agranular cortical profiles. By showing an association between the shared dimension and this microstructural gradient, we confirm an overall heightened susceptibility of paralimbic cortices to disease-related cortical thickness changes. Several architectonic features of the paralimbic cortices may underscore their increased susceptibility to disease-related effects. On the one hand, these regions have an architecture that may permit an increased potential for brain plasticity. This includes an overall reduced neuronal density in paralimbic regions compared to eulaminate cortices that may be more permissive for dendritic arborization and synaptogenesis [34]. Paralimbic areas also express several developmental markers into adulthood that cease to be expressed in other areas after ontogeny, such as growth associated protein GAP-43 [79]. On the other hand, limbic areas are known to have a relatively late myelination compared to sensory/motor areas and lower overall myelin content in adults. The role of intracortical myelination in plasticity is likely to be complex, but several streams of evidence point to a role of myelin acting as a buffer against plasticity. In addition to acting as an insulator for electrical transmission, myelin associated growth inhibitors limit activity and experience-induced axon sprouting, with downstream effects of synaptic plasticity [80]. The reduced myelin content, together with increased complexity of dendritic arborization in transmodal and paralimbic regions may render cortical microstructure in these regions more susceptible to pathological alterations, which would echo observations in other neurological conditions. For example, the core pathological substrates of drug-resistant temporal epilepsy is thought to be localized in limbic/paralimbic regions [81-83], and prior work has suggested rather specific changes in myelin and microstructural proxies in these areas [84, 85]. Similar findings have been observed in degenerative conditions such as Alzheimer's disease [44, 86, 87] as well as depression [88] and autism [89, 90], where pathology spreads from disease epicenters in paralimbic allocortices to invade more widespread cortical/subcortical networks. These findings collectively show that cellular and molecular features of paralimbic cortices and their cortico-cortical pathways promote brain plasticity as well as higher metabolic activity, and are thus likely more vulnerable to both developmental as well as acquired disruptions than other regions, supporting the hypothesis that their cortical type predisposes to a heightened vulnerability for an impact of neuropsychiatric conditions on alterations in brain morphology [34]. 
Park et al. | Cross-condition effects of major psychiatric conditions

Studying the post mortem 3D BigBrain [60], we obtained supporting confirmation for the above association between cortical microstructure and disease related susceptibility. In particular, we discovered similarly marked associations between the shared disease dimension and laminar profile skewness as well as externopyramidization, both features assessing depth-dependent shifts in the distribution in cell densities [38, 68]. In prior work, we reported that the profile skewness feature discriminates unimodal granular cortices from agranular/dysgranular paralimbic regions at a cortexwide level [65], and also helped to delineate the iso-to-allocortical axis in the mesiotemporal lobe system [66]. Studying typical adolescent development, changes in profile skewness of myelinsensitive MRI contrasts have furthermore been reported to spatially co-localize with expression patterns of genes enriched in oligodendrocytes [65]. As a complementary feature of laminar organization, externopyramidization classically contextualizes the ratio of neuronal densities between supragranular and infragranular cortical layers. It increases when the cortex is cytoarchitectonically more differentiated, which happens in primary areas with a marked layer 4 [68]. Thus, the association of these cortical depth-dependent cytoarchitectural features with the shared disease effect confirms the in vivo findings with ultrahigh resolution cytoarchitecture data suggesting that paralimbic areas, sensitive to transdiagnostic cortical alterations, are less laminarly differentiated. Furthermore, prior cellular and transcriptomic studies indicate regional susceptibility of synaptic elements as well as mutated genes in schizophrenia [91, 92] and bipolar disorder [93]. Indeed, major depression may cause atrophy of neurons in limbic regions [94], pointing histopathological susceptibility of paralimbic areas in psychiatric conditions.

We also observed a marginal association between the transdiagnostic effect on brain structure and the principal functional connectivity gradient, but findings were overall weaker than for the above in vivo and post mortem derived microstructural gradients. Motifs of macroscale intrinsic functional connectivity also show an overall sensory-fugal pattern [40, 95-97], but the associated gradients generally run from sensory/motor towards more heteromodal association cortices such as the default mode and frontoparietal networks, and not the paralimbic regions. These findings may indirectly support the conclusion that transdiagnostic disease effects on brain morphology may more closely align with spatial trends in microstructure rather than with macroscale functional differentiation. As brain organization show functional heterogeneity and multiplicity, investigation of associations between the transdiagnostic effects and multiple functional gradients is required for further studies. Notably, however, the cortical morphological data from the ENIGMA dataset were only available in the Desikan-Killany parcellation [62], a relatively macroscopic scheme mainly based on sulco-gyral features. In addition to not offering a high granularity on cortical arealization, the reliance on folding alone may only provide rather limited sensitivity to contextualize our findings with respect to functional topographies. It would thus be relevant to re-evaluate functional gradient association based on functionally-defined parcellations $[98,99]$ or at a vertex-level.

In addition to our findings showing overall associations between the transdiagnostic effect and sensory-fugal microstructural gradients, we observed associations to the spatial distribution of different neurotransmitter systems derived from in vivo neuroimaging. Notably, associations were seen both to serotonin (5-HT1a and 5-HT1b) and dopamine receptors and transporters (DAT/D1 and D2), two important markers of mental health and targets for pharmacological treatments [100-108]. In both cases (i.e. 5-HT1a vs 5-HT1b, DAT/D1 vs D2), associations to the disease effect were of opposite polarity, confirming prior work in rodents [109-113] and humans [114-117]. Associations with in vivo neurotransmitter topographies provide a novel way of indirectly assessing the relationship between shared abnormalities on cortical morphology and neurotransmitter systems so that we can understand putative mechanisms of shared morphological abnormalities, extending prior work from 
Park et al. | Cross-condition effects of major psychiatric conditions

rodents and humans. As an integrative analysis, we opted for supervised machine learning to predict the shared disease effect. This analysis revealed that not a single feature, but rather combinations of both microstructure and dopamine/serotonin transmitter systems have highest utility in predicting the spatial pattern of the transdiagnostic morphological dimensions. Overall, our findings add new evidence for a principal organizational dimension that differentiates sensory-motor networks from transmodal cortices in typical human brain organization [37, 39, 118], and furthermore describes the main axis of cortex-wide susceptibility to transdiagnostic effects of common mental health conditions. Altogether, the observed associations between multiscale neural mechanisms and transdiagnostic anomalies of cortical morphology provide a potentially integrative framework for understanding neuropathology in psychiatry and the development of treatment that cut across traditional disease boundaries. 
bioRxiv preprint doi: https://doi.org/10.1101/2021.10.29.466434; this version posted November 2,2021 . The copyright holder for this preprint (which was not certified by peer review) is the author/funder, who has granted bioRxiv a license to display the preprint in perpetuity. It is made available under aCC-BY-NC-ND 4.0 International license.

Park et al. | Cross-condition effects of major psychiatric conditions

\section{METHODS}

\section{Study dataset}

a) ENIGMA data: We analyzed T1-weighted data from people with a diagnosis of $(\mathrm{n}=12,876)$ ASD $(\mathrm{n}=1,821)$, ADHD $(\mathrm{n}=1,815), \operatorname{MDD}(\mathrm{n}=2,695)$, OCD $(\mathrm{n}=2,274), \operatorname{BD}(\mathrm{n}=1,555)$, and SZ $(\mathrm{n}=$ $2,716)$ and site matched healthy controls $(n=15,670)$ from 145 independent cohorts participating in prior ENIGMA consortium studies [26-31]. Demographic information is summarized in Table S1 and available in a recent cross-condition study [32]. Data from each center were processed using the standard ENIGMA workflow (http://enigma.ini.usc.edu/protocols/imaging-protocols/). Processing was conducted using FreeSurfer [119-121] that involves magnetic field inhomogeneity correction, non-brain tissue removal, intensity normalization, and tissue segmentation. Estimated white and pial surfaces were inflated to spheres and registered to the fsaverage template. Based on the DesikanKilliany atlas [62], cortical thickness was measured for 68 gray matter brain regions. For each psychiatric condition, the ENIGMA groups performed multiple linear regression analyses to fit cortical thickness measures with age, age squared, sex, and site information. The meta-analytic profiles of between-group differences between patients and controls were estimated via an inverse variance-weighted random-effects model, which can be obtained from the previous study [32] (Fig. 1A). If the studies provided multiple effect sizes across children/adolescents/adults, only the effects from the adult sample were used, in order to match the age range across conditions. The positive/negative effects indicate increases/decreases in cortical thickness in patients relative to controls. Individual cohort investigators obtained approval from local institutional ethics boards, and informed consent was obtained from study participants or their guardians.

b) HCP data: To generate microstructural and functional connectivity gradients, we also studied 207 unrelated healthy young adults $(60 \%$ females, mean age $\pm \mathrm{SD}=28.73 \pm 3.73$ years $)$ from the HCP dataset [59]. In the HCP, multimodal imaging data comprising T1- and T2-weighted as well as rsfMRI were acquired on a Siemens Skyra 3T at Washington University. The cohort selection is identical to our prior work $[61,122]$. T1-weighted images were acquired using a magnetizationprepared rapid gradient echo (MPRAGE) sequence (repetition time (TR) $=2,400 \mathrm{~ms}$; echo time (TE) $=2.14 \mathrm{~ms}$; inversion time $(\mathrm{TI})=1,000 \mathrm{~ms}$; flip angle $=8^{\circ}$; field of view $(\mathrm{FOV})=224 \times 224 \mathrm{~mm}^{2}$; voxel size $=0.7 \mathrm{~mm}$ isotropic; 256 slices). T2-weighted data were obtained using a T2-SPACE sequence, with the same acquisition parameters as for the T1-weighted data except for TR (3,200 ms), TE (565 ms), and flip angle (variable). The rs-fMRI data were collected using a gradient-echo echoplanar imaging sequence $\left(\mathrm{TR}=720 \mathrm{~ms} ; \mathrm{TE}=33.1 \mathrm{~ms}\right.$; flip angle $=52^{\circ} ; \mathrm{FOV}=208 \times 180 \mathrm{~mm}^{2}$; voxel size $=2 \mathrm{~mm}$ isotropic; number of slices $=72$; and 1,200 volumes per time series), where participants were instructed to keep their eyes open looking at a fixation cross during the scan. Two sessions (left-to-right and right-to-left phase-encoded directions) of rs-fMRI data were acquired, providing up to four time series per participant.

Images underwent minimal preprocessing pipelines using FSL, FreeSurfer, and Workbench as follows [123-125]:

i) T1- and T2-weighted data: Data were corrected for gradient nonlinearity and b0 distortions, and then T1- and T2-weighted data were co-registered using a rigid-body transformation. Bias field was adjusted based on the inverse intensities from the T1- and T2-weighting. The white and pial surfaces were generated [119-121], and the mid-thickness surface was generated by averaging them. The midthickness surface was inflated and the spherical surface was registered to the Conte6 9 template with $164 \mathrm{k}$ vertices [126] using MSMAll [99] and downsampled to a 32k vertex mesh.

ii) Microstructure data: Myelin-sensitive proxy was estimated based on the ratio of the T1- and T2weighted contrast $[127,128]$. We generated 14 equivolumetric surfaces within the cortex and sampled T1w/T2w intensity along these surfaces [37]. A microstructural similarity matrix was constructed by 
Park et al. | Cross-condition effects of major psychiatric conditions

calculating linear correlation of cortical depth-dependent $\mathrm{T} 1 \mathrm{w} / \mathrm{T} 2 \mathrm{w}$ intensity profiles between different cortical regions based on the Desikan-Killiany atlas [62], controlling for the average wholecortex intensity profile [37]. The matrix was thresholded at zero and log-transformed [37]. A group matrix was constructed by averaging matrices across participants.

iii) $r s$-fMRI data: Data were corrected for distortions and head motion, and registered to the T1weighted data and subsequently to MNI152 standard space. Magnetic field bias correction, skull removal, and intensity normalization were performed. Noise components attributed to head movement, white matter, cardiac pulsation, arterial, and large vein related contributions were removed using FMRIB's ICA-based X-noiseifier (ICA-FIX) [129]. Preprocessed time series were mapped to the standard 'grayordinate' space using a cortical ribbon-constrained volume-to-surface mapping algorithm. The total mean of the time series of each left-to-right/right-to-left phase-encoded data was subtracted to adjust the discontinuity between the two datasets and then concatenated to form a single time series. A functional connectivity matrix was constructed by calculating the linear time series correlations between Desikan-Killiany parcels [62], followed by Fisher's r-to-z transformation [130]. Individual connectivity matrices were averaged to construct a group level connectome.

\section{Shared effects of cortical thickness differences across conditions}

To assess transdiagnostic effects of cortical thickness differences in patients relative to controls, we applied principal component analysis to the concatenated effect size maps across six conditions [131] (Fig. 1B and Fig. S1A). The first principal dimension was determined as the shared disease effect. We summarized the effects according to seven intrinsic functional communities [63], as well as four cortical hierarchical levels [40]. We additionally calculated mean effect size across the conditions to intuitively interpret shared disease effect (Fig. S1B), and also estimated principal dimension based on the data sourced from the ENIGMA toolbox (i.e., Cohen's d; Fig. S1C). We compared the shared dimension and the effect size of each condition via linear correlations to assess the degree of contribution of each condition (Fig. S2). The significance of the correlation was determined using 1,000 non-parametric spin-tests, to account for spatial autocorrelation [132], and corrected for multiple comparisons using a FDR procedure [133]. To assess robustness, we performed leave-onecondition-out cross-validation. Specifically, we estimated the shared dimension using five conditions by excepting for a single condition, and assessed similarity with the shared disease effect estimated based on the whole six conditions (Fig. S3). We calculated significance of the correlation using 1,000 spin-tests, and multiple comparisons were corrected using FDR [132, 133].

\section{Associations to microstructural and functional connectivity gradients}

We evaluated the underlying connectome organizations of the shared disease effects. Based on T1w/T2w and rs-fMRI data obtained from the HCP database [59], we estimated microstructural and functional gradients, the low dimensional representation of connectome organizations explaining spatial variation in the connectome data [37, 39], using BrainSpace (https://github.com/MICA-

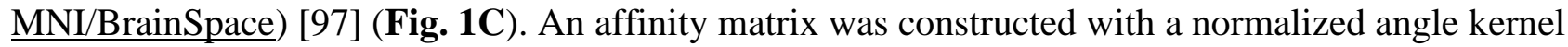
from the group averaged connectivity matrix with the top $10 \%$ entries for each parcel. The connectome gradients were estimated using diffusion map embedding [134], which is robust to noise and computationally efficient compared to other non-linear manifold learning techniques [71, 135]. It is controlled by two parameters $\alpha$ and t, where $\alpha$ controls the influence of the density of sampling points on the manifold ( $\alpha=0$, maximal influence; $\alpha=1$, no influence) and t scales eigenvalues of the diffusion operator. The parameters were set as $\alpha=0.5$ and $t=0$ to retain the global relations between data points in the embedded space, following prior applications [37, 39, 47, 97, 136]. We associated the shared effect with these gradients using linear correlation (Fig. 1D), where the significance was 
Park et al. | Cross-condition effects of major psychiatric conditions

assessed using 1,000 spin-tests followed by FDR [132, 133].

\section{Cytoarchitectonic associations with shared disease effects}

We aimed to associate the shared dimensions with histology-driven cytoarchitectonic features derived from BigBrain surfaces with 62 cortical areas (https://bigbrain.loris.ca/main.php) [60]. Specifically, BigBrain is a ultra-high resolution, 3D volumetric reconstruction of a post mortem Merker-stained and sliced human brain from a 65-year-old male, with specialized pial and white matter surface reconstructions [60]. The post mortem brain was paraffin-embedded, coronally sliced into 740020 $\mu \mathrm{m}$ sections, silver-stained for cell bodies [137], and digitized. A 3D reconstruction was implemented with a successive coarse-to-fine hierarchical procedure, resulting in a full brain volume. Among 68 regions defined by the Desikan-Killiany atlas [62], three regions per hemisphere, including banks of superior temporal sulcus, frontal pole, and temporal pole, were excluded as the BigBrain did not provide data for these regions. We generated 18 equivolumetric cortical surfaces within the cortex (https://github.com/caseypaquola/BigBrainWarp) and sampled the intensity values along these surfaces. Based on the intensity values, we calculated four moment features, including mean, SD, skewness, and kurtosis, as well as externopyramidization (Fig. 2A-B). The mean and SD represent the overall intensity distribution of cytoarchitecture across layers, skewness indicates shifts in intensity values towards supragranular layers (i.e., positive skewness) or flat distribution (i.e., negative skewness), and kurtosis identifies whether the tails of the intensity distribution contain extreme values. Externopyramidization reflects gradual shifts of intensity values from infragranular to supragranular layers defined as follows [69]:

$$
\text { Externopyramidization }=\frac{\text { (intensity) }}{\text { mean(intensity) }} \times \frac{1-\text { thickness }_{\text {supra }}}{\text { thickness }_{\text {total }}}
$$

To assess associations with shared disease effects, we calculated linear correlations between cytoarchitectonic features and shared effects (Fig. 2C). The significance of the correlations was assessed using 1,000 spin-tests followed by FDR across different cytoarchitectonic features [132, 133].

\section{Associations between transmitter systems and shared effects}

To provide underlying molecular properties of the shared effects in neuroanatomical disruptions across different psychiatric conditions, we associated the shared dimensions with ten different neurotransmitter maps of healthy controls provided by prior independent PET/SPECT studies [5358], which contain neurotransmitters of FDOPA, GABAa, transporters of DAT, NAT, SERT, and receptors of D1, D2, 5-HT1a, 5-HT1b, and 5-HT2a (https://github.com/juryxy/JuSpace) [52] (Fig. 3A). All PET maps were linearly rescaled to have intensity values between 0 and 100 [52]. After mapping the neurotransmitter maps onto the Desikan-Killiany atlas [62], we calculated linear correlations between the shared effects and each neurotransmitter map (Fig. 3B), and assessed the significance using 1,000 spin tests followed by FDR to adjust for multiple comparisons across ten different maps [132, 133].

\section{Prediction of shared effects using multiscale features}

We associated multiscale features and shared effects using supervised machine learning to incorporate our findings (Fig. 4). Specifically, we aimed to predict the shared disease effects using concatenated multiscale features of microstructural and functional gradients, cytoarchitectonic (i.e., mean, SD, skewness, kurtosis, externopyramidization), and transmitter maps (i.e., D1, D2, 5-HT1a, 5-HT1b, 5-HT2a, FDOPA, GABAa, DAT, NAT, SERT). We used five-fold nested cross-validation [72-74] with LASSO regression [70]. Nested cross-validation split the dataset into training (4/5) and 
Park et al. | Cross-condition effects of major psychiatric conditions

test (1/5) partitions, and each training partition was further split into inner training and testing folds using another five-fold cross-validation. The model with the best performance (lowest MAE) across the inner folds was applied to the test partition of the outer fold. Among the multiscale features, we selected performant features using LASSO regularization, and the effect size was predicted using linear regression with the selected features. The procedure was repeated 100 times with different training and test partitions. Prediction accuracy was evaluated with linear correlations between the actual and predicted effect size and the MAE, with their $95 \%$ confidence interval. Permutation-based correlations across 1,000 tests were conducted by randomly shuffling cortical regions to verify whether the prediction performance exceeded chance levels. We also performed the prediction analysis using the effect size of each condition (Fig. S4). 
Park et al. | Cross-condition effects of major psychiatric conditions

\section{ACKNOWLEDGMENTS}

The authors would like to express their gratitude to the open science initiatives that made this work possible: (i) The ENIGMA-Epilepsy consortium and in particular the ASD, ADHD, MDD, OCD, BD, and SZ working groups (http://enigma.ini.usc.edu/ongoing/) and (ii) The Human Connectome Project (Principal Investigators: David Van Essen and Kamil Ugurbil; 1U54MH091657) funded by the 16 NIH Institutes and Centers that support the NIH Blueprint for Neuroscience Research; and by the McDonnell Center for Systems Neuroscience at Washington University.

\section{FUNDING}

Bo-yong Park was funded by the National Research Foundation of Korea (NRF2021R1F1A1052303), Institute for Information and Communications Technology Planning and Evaluation (IITP) funded by the Korea Government (MSIT) (2020-0-01389, Artificial Intelligence Convergence Research Center, Inha University; 2021-0-02068, Artificial Intelligence Innovation Hub), and Institute for Basic Science (IBS-R015-D1). Valeria Kebets was funded by the Quebec Autism Research Training Fellowship of the Transforming Autism Care Consortium (TACC). Sara Larivière was funded by the Canadian Institutes of Health Research (CIHR). Meike D. Hettwer was funded by the Max Planck society and the German Federal Ministry of Education and Research (BMBF). Martine Hoogman is supported by a personal Veni grant from the Netherlands Organization for Scientific Research (NWO, grant number 91619115). Lianne Schmaal was funded by NHMRC Career Development Fellowship (1140764), National Institute of Mental Health of the National Institutes of Health R01MH117601. Ole A. Andreassen was funded by Research Council of Norway (\#223273, \#283798), KG Jebsen Stiftelsen. Dan J Stein is funded by the SA MRC. Dr. Matthias Kirschner acknowledges support from the National Bank Fellowship (McGill University) and the Swiss National Foundation (P2SKP3_178175). Jessica Turner was funded by National Institutes of Health (NIH 5R01MH121246). Paul Thompson and Sophia Thomopoulos are funded in part by the U.S. National Institutes of Health, under grants R01MH116147, R01MH111671, R01AG058854, RF1MH123163 and U54 EB020403. Boris C. Bernhardt acknowledges research support from the National Science and Engineering Research Council of Canada (NSERC Discovery-1304413), the CIHR (FDN-154298, PJT), SickKids Foundation (NI17-039), Azrieli Center for Autism Research (ACAR-TACC), BrainCanada, Fonds de la Recherche du Québec - Santé (FRQ-S), and the Tier-2 Canada Research Chairs program. Alan Evans and Boris C. Bernhardt were furthermore supported by the Helmholtz International BigBrain Analytics and Learning Laboratory (HiBALL).

\section{CONFLICT OF INTEREST}

Ole A. Andreassen received speaker's honorarium from Lundbeck and Sunovion, Consultant to HealthLytix. Paul M. Thompson received grant support from Biogen, Inc., and consulting payments from Kairos Venture Capital, for work unrelated to the current manuscript. Other authors declare no conflicts of interest. 
Park et al. | Cross-condition effects of major psychiatric conditions

\section{REFERENCES}

1. Whiteford HA, Degenhardt L, Rehm J, Baxter AJ, Ferrari AJ, Erskine HE, et al. Global burden of disease attributable to mental and substance use disorders: Findings from the Global Burden of Disease Study 2010. Lancet. 2013;382:1575-1586.

2. American Psychiatric Association. Diagnostic and Statistical Manual of Mental Disorders. 5th ed. American Psychiatric Press, Washington, DC; 2013.

3. Marshall M. The hidden links between mental disorders. Nature. 2020;581:19-21.

4. Xia CH, Ma Z, Ciric R, Gu S, Betzel RF, Kaczkurkin AN, et al. Linked dimensions of psychopathology and connectivity in functional brain networks. Nat Commun. 2018;9:3003.

5. Insel T, Cuthbert B, Garvey M, Heinssen R, Pine D, Quinn K, et al. Research Domain Criteria ( RDoC ): Toward a New Classification Framework for Research on Mental Disorders. Am J Psychiatry Online. 2010:748-751.

6. Cuthbert BN. The RDoC framework: facilitating transition from ICD/DSM to dimensional approaches that integrate neuroscience and psychopathology. World Psychiatry. 2014;13:2835 .

7. Kotov R, Krueger RF, Watson D. A paradigm shift in psychiatric classification: the Hierarchical Taxonomy Of Psychopathology (HiTOP). World Psychiatry. 2018;17:24-25.

8. Kotov R, Krueger RF, Watson D, Achenbach TM, Althoff RR, Bagby RM, et al. The Hierarchical Taxonomy of Psychopathology (HiTOP): A dimensional alternative to traditional nosologies. J Abnorm Psychol. 2017;126:454-477.

9. Parkes L, Moore TM, Calkins ME, Cook PA, Cieslak M, Roalf DR, et al. Transdiagnostic dimensions of psychopathology explain individuals' unique deviations from normative neurodevelopment in brain structure. Transl Psychiatry. 2021;11:232.

10. Borsboom D. A network theory of mental disorders. World Psychiatry. 2017;16:5-13.

11. Kaczkurkin AN, Park SS, Sotiras A, Moore TM, Calkins ME, Cieslak M, et al. Evidence for dissociable linkage of dimensions of psychopathology to brain structure in youths. Am J Psychiatry. 2019;176:1000-1009.

12. Kaczkurkin AN, Sotiras A, Baller EB, Barzilay R, Calkins ME, Chand GB, et al. Neurostructural Heterogeneity in Youths With Internalizing Symptoms. Biol Psychiatry. 2020;87:473-482.

13. Linden DEJ. The challenges and promise of neuroimaging in psychiatry. Neuron. 2012;73:822.

14. Hong SJ, Vogelstein JT, Gozzi A, Bernhardt BC, Yeo BTT, Milham MP, et al. Toward Neurosubtypes in Autism. Biol Psychiatry. 2020;88:111-128.

15. Castellanos FX, Di Martino A, Craddock RC, Mehta AD, Milham MP. Clinical applications of the functional connectome. Neuroimage. 2013;80:527-540.

16. Milham MP, Vogelstein J, Xu T. Removing the Reliability Bottleneck in Functional Magnetic Resonance Imaging Research to Achieve Clinical Utility. JAMA Psychiatry. 2021;39:4213-4227. 
Park et al. | Cross-condition effects of major psychiatric conditions

17. Valk SL, Di Martino A, Milham MP, Bernhardt BC. Multicenter mapping of structural network alterations in autism. Hum Brain Mapp. 2015;36:2364-2373.

18. Hong SJ, Valk SL, Di Martino A, Milham MP, Bernhardt BC. Multidimensional neuroanatomical subtyping of autism spectrum disorder. Cereb Cortex. 2018;28:3578-3588.

19. Hajek T, Carrey N, Alda M. Neuroanatomical abnormalities as risk factors for bipolar disorder. Bipolar Disord. 2005;7:393-403.

20. Khundrakpam BS, Lewis JD, Kostopoulos P, Carbonell F, Evans AC. Cortical Thickness Abnormalities in Autism Spectrum Disorders Through Late Childhood, Adolescence, and Adulthood: A Large-Scale MRI Study. Cereb Cortex. 2017;27:1721-1731.

21. Lorenzetti V, Allen NB, Fornito A, Yücel M. Structural brain abnormalities in major depressive disorder: A selective review of recent MRI studies. J Affect Disord. 2009;117:117.

22. Wise T, Radua J, Via E, Cardoner N, Abe O, Adams TM, et al. Common and distinct patterns of grey-matter volume alteration in major depression and bipolar disorder: Evidence from voxel-based meta-analysis. Mol Psychiatry. 2017;22:1455-1463.

23. Goodkind M, Eickhoff SB, Oathes DJ, Jiang Y, Chang A, Jones-Hagata LB, et al. Identification of a common neurobiological substrate for mental Illness. JAMA Psychiatry. 2015;72:305-315.

24. Gong Q, Scarpazza C, Dai J, He M, Xu X, Shi Y, et al. A transdiagnostic neuroanatomical signature of psychiatric illness. Neuropsychopharmacology. 2019;44:869-875.

25. Thompson PM, Jahanshad N, Ching CRK, Salminen LE, Thomopoulos SI, Bright J, et al. ENIGMA and global neuroscience: A decade of large-scale studies of the brain in health and disease across more than 40 countries. Transl Psychiatry. 2020;10:100.

26. van Rooij D, Anagnostou E, Arango C, Auzias G, Behrmann M, Busatto GF, et al. Cortical and subcortical brain morphometry differences between patients with autism spectrum disorder and healthy individuals across the lifespan: Results from the ENIGMA ASD working group. Am J Psychiatry. 2018;175:359-369.

27. Hoogman M, Muetzel R, Guimaraes JP, Shumskaya E, Mennes M, Zwiers MP, et al. Brain imaging of the cortex in ADHD: A coordinated analysis of large-scale clinical and population-based samples. Am J Psychiatry. 2019;176:531-542.

28. Schmaal L, Hibar DP, Sämann PG, Hall GB, Baune BT, Jahanshad N, et al. Cortical abnormalities in adults and adolescents with major depression based on brain scans from 20 cohorts worldwide in the ENIGMA Major Depressive Disorder Working Group. Mol Psychiatry. 2017;22:900-909.

29. Boedhoe PSW, Schmaal L, Abe Y, Alonso P, Ameis SH, Anticevic A, et al. Cortical abnormalities associated with pediatric and adult obsessive-compulsive disorder: Findings from the ENIGMA obsessive-compulsive disorder working group. Am J Psychiatry. 2018; $175: 453-462$.

30. Hibar DP, Westlye LT, Doan NT, Jahanshad N, Cheung JW, Ching CRK, et al. Cortical abnormalities in bipolar disorder: An MRI analysis of 6503 individuals from the ENIGMA Bipolar Disorder Working Group. Mol Psychiatry. 2018;23:932-942.

31. van Erp TGM, Walton E, Hibar DP, Schmaal L, Jiang W, Glahn DC, et al. Cortical Brain 
Park et al. | Cross-condition effects of major psychiatric conditions

Abnormalities in 4474 Individuals With Schizophrenia and 5098 Control Subjects via the Enhancing Neuro Imaging Genetics Through Meta Analysis (ENIGMA) Consortium. Biol Psychiatry. 2018;84:644-654.

32. Patel Y, Parker N, Shin J, Howard D, French L, Thomopoulos SI, et al. Virtual Histology of Cortical Thickness and Shared Neurobiology in 6 Psychiatric Disorders. JAMA Psychiatry. 2021;78:47.

33. Opel N, Goltermann J, Hermesdorf M, Berger K, Baune BT, Dannlowski U. Cross-Disorder Analysis of Brain Structural Abnormalities in Six Major Psychiatric Disorders : A Secondary Analysis of Mega- and Meta- analytical Findings From the ENIGMA Consortium. Biol Psychiatry. 2020;88:678-686.

34. García-Cabezas MÁ, Zikopoulos B, Barbas H. The Structural Model: a theory linking connections, plasticity, pathology, development and evolution of the cerebral cortex. Brain Struct Funct. 2019;224:985-1008.

35. Amunts K, Mohlberg H, Bludau S, Zilles K. Julich-Brain: A 3D probabilistic atlas of the human brain's cytoarchitecture. Science (80- ). 2020;369:988-992.

36. Wagstyl K, Lepage C, Bludau S, Zilles K, Fletcher PC, Amunts K, et al. Mapping cortical laminar structure in the 3D bigbrain. Cereb Cortex. 2018;28:2551-2562.

37. Paquola C, Vos De Wael R, Wagstyl K, Bethlehem RAI, Hong SJ, Seidlitz J, et al. Microstructural and functional gradients are increasingly dissociated in transmodal cortices. PLoS Biol. 2019;17:e3000284.

38. Schleicher A, Morosan P, Amunts K, Zilles K. Quantitative architectural analysis: A new approach to cortical mapping. J Autism Dev Disord. 2009;39:1568-1581.

39. Margulies DS, Ghosh SS, Goulas A, Falkiewicz M, Huntenburg JM, Langs G, et al. Situating the default-mode network along a principal gradient of macroscale cortical organization. Proc Natl Acad Sci U S A. 2016;113:12574-12579.

40. Mesulam MM. From sensation to cognition. Brain. 1998;121:1013-1052.

41. Sanides F. Die Architektonik des Menschlichen Stirnhirns. vol. 98. Berlin, Heidelberg: Springer Berlin Heidelberg; 1962.

42. Huntenburg JM, Bazin PL, Margulies DS. Large-Scale Gradients in Human Cortical Organization. Trends Cogn Sci. 2018;22:21-31.

43. Goulas A, Changeux JP, Wagstyl K, Amunts K, Palomero-Gallagher N, Hilgetag CC. The natural axis of transmitter receptor distribution in the human cerebral cortex. Proc Natl Acad Sci U S A. 2021;118:e2020574118.

44. Vogel JW, La Joie R, Grothe MJ, Diaz-Papkovich A, Doyle A, Vachon-Presseau E, et al. A molecular gradient along the longitudinal axis of the human hippocampus informs large-scale behavioral systems. Nat Commun. 2020;11:960.

45. Valk SL, Xu T, Margulies DS, Masouleh SK, Paquola C, Goulas A, et al. Shaping brain structure: Genetic and phylogenetic axes of macroscale organization of cortical thickness. Sci Adv. 2020;6:1-15.

46. Vainik U, Paquola C, Wang X, Zheng Y, Bernhardt B, Misic B, et al. Heritability of cortical morphology reflects a sensory-fugal plasticity gradient. BioRxiv. 2020. 2020. 
Park et al. | Cross-condition effects of major psychiatric conditions

47. Hong S-J, Vos De Wael R, Bethlehem RAI, Lariviere S, Paquola C, Valk SL, et al. Atypical functional connectome hierarchy in autism. Nat Commun. 2019;10:1022.

48. Lowe AJ, Paquola C, Vos de Wael R, Girn M, Lariviere S, Tavakol S, et al. Targeting agerelated differences in brain and cognition with multimodal imaging and connectome topography profiling. Hum Brain Mapp. 2019;40:5213-5230.

49. Bijsterbosch J, Harrison SJ, Jbabdi S, Woolrich M, Beckmann C, Smith S, et al. Challenges and future directions for representations of functional brain organization. Nat Neurosci. 2020;23:1484-1495.

50. Haak K V., Marquand AF, Beckmann CF. Connectopic mapping with resting-state fMRI. Neuroimage. 2018;170:83-94.

51. Mars RB, Sotiropoulos SN, Passingham RE, Sallet J, Verhagen L, Khrapitchev AA, et al. Whole brain comparative anatomy using connectivity blueprints. Elife. 2018;7:1-15.

52. Dukart J, Holiga S, Rullmann M, Lanzenberger R, Hawkins PCT, Mehta MA, et al. JuSpace: A tool for spatial correlation analyses of magnetic resonance imaging data with nuclear imaging derived neurotransmitter maps. Hum Brain Mapp. 2021;42:555-566.

53. Alakurtti K, Johansson JJ, Joutsa J, Laine M, Bäckman L, Nyberg L, et al. Long-term testretest reliability of striatal and extrastriatal dopamine D2/3 receptor binding: Study with [11C]raclopride and high-resolution PET. J Cereb Blood Flow Metab. 2015;35:1199-1205.

54. Hesse S, Becker GA, Rullmann M, Bresch A, Luthardt J, Hankir MK, et al. Central noradrenaline transporter availability in highly obese, non-depressed individuals. Eur J Nucl Med Mol Imaging. 2017;44:1056-1064.

55. Kaller S, Rullmann M, Patt M, Becker GA, Luthardt J, Girbardt J, et al. Test-retest measurements of dopamine D1-type receptors using simultaneous PET/MRI imaging. Eur J Nucl Med Mol Imaging. 2017;44:1025-1032.

56. Savli M, Bauer A, Mitterhauser M, Ding YS, Hahn A, Kroll T, et al. Normative database of the serotonergic system in healthy subjects using multi-tracer PET. Neuroimage. 2012;63:447-459.

57. García Gómez F, Huertas I, Lojo Ramírez J, García Solís D. Elaboración de una plantilla de SPM para la normalización de imágenes de PET con 18F-DOPA. Imagen Diagnóstica. 2018;9:23-25.

58. Dukart J, Holiga Š, Chatham C, Hawkins P, Forsyth A, McMillan R, et al. Cerebral blood flow predicts differential neurotransmitter activity. Sci Rep. 2018;8:4074.

59. Van Essen DC, Smith SM, Barch DM, Behrens TEJ, Yacoub E, Ugurbil K. The WU-Minn Human Connectome Project: an overview. Neuroimage. 2013;80:62-79.

60. Amunts K, Lepage C, Borgeat L, Mohlberg H, Dickscheid T, Rousseau MÉ, et al. BigBrain: An ultrahigh-resolution 3D human brain model. Science (80- ). 2013;340:1472-1475.

61. Larivière S, Paquola C, Park B, Royer J, Wang Y, Benkarim O, et al. The ENIGMA Toolbox: multiscale neural contextualization of multisite neuroimaging datasets. Nat Methods. 2021. 30 June 2021. https://doi.org/10.1038/s41592-021-01186-4.

62. Desikan RS, Ségonne F, Fischl B, Quinn BT, Dickerson BC, Blacker D, et al. An automated labeling system for subdividing the human cerebral cortex on MRI scans into gyral based 
Park et al. | Cross-condition effects of major psychiatric conditions

regions of interest. Neuroimage. 2006;31:968-980.

63. Buckner RL, Krienen FM, Castellanos A, Diaz JC, Yeo BTT. The organization of the human cerebellum estimated by intrinsic functional connectivity. J Neurophysiol. 2011;106:23222345.

64. Paquola C, Royer J, Lewis LB, Lepage C, Glatard T, Wagstyl K, et al. The BigBrainWarp toolbox for integration of BigBrain 3D histology with multimodal neuroimaging. Elife. 2021;10:e70119.

65. Paquola C, Bethlehem RA, Seidlitz J, Wagstyl K, Romero-Garcia R, Whitaker KJ, et al. Shifts in myeloarchitecture characterise adolescent development of cortical gradients. Elife. 2019;8:e50482.

66. Paquola C, Benkarim O, DeKraker J, Larivière S, Frässle S, Royer J, et al. Convergence of cortical types and functional motifs in the human mesiotemporal lobe. Elife. 2020;9:e60673.

67. Royer J, Paquola C, Larivière S, Vos de Wael R, Tavakol S, Lowe AJ, et al.

Myeloarchitecture gradients in the human insula: Histological underpinnings and association to intrinsic functional connectivity. Neuroimage. 2020;216:116859.

68. Goulas A, Zilles K, Hilgetag CC. Cortical Gradients and Laminar Projections in Mammals. Trends Neurosci. 2018;41:775-788.

69. Paquola C, Seidlitz J, Benkarim O, Royer J, Klimes P, Bethlehem RAI, et al. A multi-scale cortical wiring space links cellular architecture and functional dynamics in the human brain. vol. 18. 2020.

70. Tibshirani R. Regression Shrinkage and Selection Via the Lasso. J R Stat Soc Ser B. 1996;58:267-288.

71. Tenenbaum JB, Silva V de, Langford JC. A Global Geometric Framework for Nonlinear Dimensionality Reduction. Science (80- ). 2000;290:2319-2323.

72. Varma S, Simon R. Bias in error estimation when using cross-validation for model selection. BMC Bioinformatics. 2006;7:91.

73. Cawley GC, Talbot NLC. On over-fitting in model selection and subsequent selection bias in performance evaluation. J Mach Learn Res. 2010;11:2079-2107.

74. Parvandeh S, Yeh HW, Paulus MP, McKinney BA. Consensus features nested crossvalidation. Bioinformatics. 2020;36:3093-3098.

75. Bedford SA, Park MTM, Devenyi GA, Tullo S, Germann J, Patel R, et al. Large-scale analyses of the relationship between sex, age and intelligence quotient heterogeneity and cortical morphometry in autism spectrum disorder. Mol Psychiatry. 2020;25:614-628.

76. Markello R, Shafiei G, Zheng Y-Q, Mišić B. abagen: A toolbox for the Allen Brain Atlas genetics data. Zenodo. 2020. 2020. https://doi.org/http://doi.org/10.5281/zenodo.4091537.

77. Hawrylycz MJ, Lein ES, Guillozet-Bongaarts AL, Shen EH, Ng L, Miller JA, et al. An anatomically comprehensive atlas of the adult human brain transcriptome. Nature. 2012;489:391-399.

78. Arnatkeviciute A, Fulcher BD, Fornito A. A practical guide to linking brain-wide gene expression and neuroimaging data. Neuroimage. 2019;189:353-367. 
Park et al. | Cross-condition effects of major psychiatric conditions

79. Benowitz LI, Routtenberg A. GAP-43: an intrinsic determinant of neuronal development and plasticity. Trends Neurosci. 1997;20:84-91.

80. Dinse J, Härtwich N, Waehnert MD, Tardif CL, Schäfer A, Geyer S, et al. A cytoarchitecture-driven myelin model reveals area-specific signatures in human primary and secondary areas using ultra-high resolution in-vivo brain MRI. Neuroimage. 2015;114:7187.

81. Thom M. Review: Hippocampal sclerosis in epilepsy: A neuropathology review. Neuropathol Appl Neurobiol. 2014;40:520-543.

82. Bernhardt BC, Bernasconi A, Liu M, Hong SJ, Caldairou B, Goubran M, et al. The spectrum of structural and functional imaging abnormalities in temporal lobe epilepsy. Ann Neurol. 2016;80:142-153.

83. Blümcke I, Thom M, Aronica E, Armstrong DD, Bartolomei F, Bernasconi A, et al. International consensus classification of hippocampal sclerosis in temporal lobe epilepsy: A Task Force report from the ILAE Commission on Diagnostic Methods. Epilepsia. 2013;54:1315-1329.

84. Adler S, Hong SJ, Liu M, Baldeweg T, Cross JH, Bernasconi A, et al. Topographic principles of cortical fluid-attenuated inversion recovery signal in temporal lobe epilepsy. Epilepsia. 2018;59:627-635.

85. Bernhardt BC, Fadaie F, Vos de Wael R, Hong SJ, Liu M, Guiot MC, et al. Preferential susceptibility of limbic cortices to microstructural damage in temporal lobe epilepsy: A quantitative T1 mapping study. Neuroimage. 2018;182:294-303.

86. La Joie R, Landeau B, Perrotin A, Bejanin A, Egret S, Pélerin A, et al. Intrinsic connectivity identifies the hippocampus as a main crossroad between alzheimer's and semantic dementiatargeted networks. Neuron. 2014;81:1417-1428.

87. Shafiei G, Markello RD, Makowski C, Talpalaru A, Kirschner M, Devenyi GA, et al. Spatial Patterning of Tissue Volume Loss in Schizophrenia Reflects Brain Network Architecture. Biol Psychiatry. 2020;87:727-735.

88. Mayberg HS, Lozano AM, Voon V, McNeely HE, Seminowicz D, Hamani C, et al. Deep brain stimulation for treatment-resistant depression. Neuron. 2005;45:651-660.

89. Zikopoulos B, García-Cabezas MÁ, Barbas H. Parallel trends in cortical gray and white matter architecture and connections in primates allow fine study of pathways in humans and reveal network disruptions in autism. vol. 16. 2018.

90. Zikopoulos B, Barbas H. Changes in prefrontal axons may disrupt the network in autism. $\mathrm{J}$ Neurosci. 2010;30:14595-14609.

91. van Berlekom AB, Muflihah CH, Snijders GJLJ, MacGillavry HD, Middeldorp J, Hol EM, et al. Synapse Pathology in Schizophrenia: A Meta-analysis of Postsynaptic Elements in Postmortem Brain Studies. Schizophr Bull. 2020;46:374-386.

92. Mizutani R, Saiga R, Takeuchi A, Uesugi K, Terada Y, Suzuki Y, et al. Three-dimensional alteration of neurites in schizophrenia. Transl Psychiatry. 2019;9.

93. Toker L, Mancarci BO, Tripathy S, Pavlidis P. Transcriptomic Evidence for Alterations in Astrocytes and Parvalbumin Interneurons in Subjects With Bipolar Disorder and Schizophrenia. Biol Psychiatry. 2018;84:787-796. 
Park et al. | Cross-condition effects of major psychiatric conditions

94. Duman RS, Sanacora G, Krystal JH. Altered Connectivity in Depression: GABA and Glutamate Neurotransmitter Deficits and Reversal by Novel Treatments. Neuron. 2019;102:75-90.

95. Burt JB, Demirtaş M, Eckner WJ, Navejar NM, Ji JL, Martin WJ, et al. Hierarchy of transcriptomic specialization across human cortex captured by structural neuroimaging topography. Nat Neurosci. 2018;21:1251-1259.

96. Vázquez-Rodríguez B, Suárez LE, Markello RD, Shafiei G, Paquola C, Hagmann P, et al. Gradients of structure-function tethering across neocortex. Proc Natl Acad Sci U S A. 2019;116:21219-21227.

97. Vos de Wael R, Benkarim O, Paquola C, Lariviere S, Royer J, Tavakol S, et al. BrainSpace: a toolbox for the analysis of macroscale gradients in neuroimaging and connectomics datasets. Commun Biol. 2020;3:103.

98. Schaefer A, Kong R, Gordon EM, Laumann TO, Zuo X-N, Holmes AJ, et al. Local-Global Parcellation of the Human Cerebral Cortex from Intrinsic Functional Connectivity MRI. Cereb Cortex. 2018;28:3095-3114.

99. Glasser MF, Coalson TS, Robinson EC, Hacker CD, Harwell J, Yacoub E, et al. A multimodal parcellation of human cerebral cortex. Nature. 2016;536:171-178.

100. Marazziti D. Understanding the role of serotonin in psychiatric diseases. F1000Research. 2017;6:180.

101. Lin SH, Lee LT, Yang YK. Serotonin and mental disorders: A concise review on molecular neuroimaging evidence. Clin Psychopharmacol Neurosci. 2014;12:196-202.

102. Lopez-Ibor JJ. The involvement of serotonin in psychiatric disorders and behaviour. Br J Psychiatry. 1988;153:26-39.

103. Conio B, Martino M, Magioncalda P, Escelsior A, Inglese M, Amore M, et al. Opposite effects of dopamine and serotonin on resting-state networks: review and implications for psychiatric disorders. Mol Psychiatry. 2020;25:82-93.

104. Volkow ND, Wang GJ, Kollins SH, Wigal TL, Newcorn JH, Telang F, et al. Evaluating dopamine reward pathway in ADHD: Clinical Implications. JAMA - J Am Med Assoc. 2009;302:1084-1091.

105. Pine A, Shiner T, Seymour B, Dolan RJ. Dopamine, time, and impulsivity in humans. J Neurosci. 2010;30:8888-8896.

106. Cropley VL, Fujita M, Innis RB, Nathan PJ. Molecular Imaging of the Dopaminergic System and its Association with Human Cognitive Function. Biol Psychiatry. 2006;59:898-907.

107. Ashok AH, Marques TR, Jauhar S, Nour MM, Goodwin GM, Young AH, et al. The dopamine hypothesis of bipolar affective disorder: The state of the art and implications for treatment. Mol Psychiatry. 2017;22:666-679.

108. Dunlop BW, Nemeroff CB. The role of dopamine in the pathophysiology of depression. Arch Gen Psychiatry. 2007;64:327-337.

109. Zhuang X, Gross C, Santarelli L, Compan V, Trillat AC, Hen R. Altered emotional states in knockout mice lacking 5-HT1A or 5-HT1B receptors. Neuropsychopharmacology. 1999;21:52S-60S. 
Park et al. | Cross-condition effects of major psychiatric conditions

110. Yohn CN, Gergues MM, Samuels BA. The role of 5-HT receptors in depression. Mol Brain. 2017;10:28.

111. Seamans JK, Gorelova N, Durstewitz D, Yang CR. Bidirectional dopamine modulation of GABAergic inhibition in prefrontal cortical pyramidal neurons. J Neurosci. 2001;21:36283638.

112. Gorelova N, Seamans JK, Yang CR. Mechanisms of dopamine activation of fast-spiking interneurons that exert inhibition in rat prefrontal cortex. J Neurophysiol. 2002;88:31503166.

113. Trantham-Davidson H, Neely LC, Lavin A, Seamans JK. Mechanisms underlying differential D1 versus D2 dopamine receptor regulation of inhibition in prefrontal cortex. J Neurosci. 2004;24:10652-10659.

114. Parsey R V., Oquendo MA, Ogden RT, Olvet DM, Simpson N, Huang YY, et al. Altered serotonin 1A binding in major depression: A [carbonyl-C-11] WAY100635 positron emission tomography study. Biol Psychiatry. 2006;59:106-113.

115. Lemonde S, Turecki G, Bakish D, Du L, Hrdina PD, Bown CD, et al. Impaired repression at a 5-hydroxytryptamine $1 \mathrm{~A}$ receptor gene polymorphism associated with major depression and suicide. J Neurosci. 2003;23:8788-8799.

116. Salatino-Oliveira A, Rohde LA, Hutz MH. The dopamine transporter role in psychiatric phenotypes. Am J Med Genet Part B Neuropsychiatr Genet. 2018;177:211-231.

117. Fernández-Jaén A, López-Martín S, Albert J, Fernández-Mayoralas DM, Fernández-Perrone AL, de La Peña MJ, et al. Cortical thickness differences in the prefrontal cortex in children and adolescents with ADHD in relation to dopamine transporter (DAT1) genotype.

Psychiatry Res - Neuroimaging. 2015;233:409-417.

118. Park B, Bethlehem RAI, Paquola C, Larivière S, Rodríguez-Cruces R, Vos de Wael R, et al. An expanding manifold in transmodal regions characterizes adolescent reconfiguration of structural connectome organization. Elife. 2021;10:e64694.

119. Fischl B, Sereno MI, Tootell RBH, Dale AM. High-resolution inter-subject averaging and a surface-based coordinate system. Hum Brain Mapp. 1999;8:272-284.

120. Dale AM, Fischl B, Sereno MI. Cortical surface-based analysis: I. Segmentation and surface reconstruction. Neuroimage. 1999;9:179-194.

121. Fischl B, Sereno MI, Dale AM. Cortical surface-based analysis: II. Inflation, flattening, and a surface-based coordinate system. Neuroimage. 1999;9:195-207.

122. Larivière S, Rodríguez-Cruces R, Royer J, Caligiuri ME, Gambardella A, Concha L, et al. Network-based atrophy modelling in the common epilepsies: A worldwide ENIGMA study. Sci Adv. 2020;6:eabc6457.

123. Glasser MF, Sotiropoulos SN, Wilson JA, Coalson TS, Fischl B, Andersson JL, et al. The minimal preprocessing pipelines for the Human Connectome Project. Neuroimage. 2013;80:105-124.

124. Jenkinson M, Beckmann CF, Behrens TEJ, Woolrich MW, Smith SM. Fsl. Neuroimage. 2012;62:782-790.

125. Fischl B. FreeSurfer. Neuroimage. 2012;62:774-781. 
Park et al. | Cross-condition effects of major psychiatric conditions

126. Van Essen DC, Glasser MF, Dierker DL, Harwell J, Coalson T. Parcellations and hemispheric asymmetries of human cerebral cortex analyzed on surface-based atlases. Cereb Cortex. 2012;22:2241-2262.

127. Glasser MF, Goyal MS, Preuss TM, Raichle ME, Van Essen DC. Trends and properties of human cerebral cortex: Correlations with cortical myelin content. Neuroimage. 2014;93:165175.

128. Glasser MF, Van Essen DC. Mapping human cortical areas in vivo based on myelin content as revealed by T1- and T2-weighted MRI. J Neurosci. 2011;31:11597-11616.

129. Salimi-Khorshidi G, Douaud G, Beckmann CF, Glasser MF, Griffanti L, Smith SM. Automatic denoising of functional MRI data: Combining independent component analysis and hierarchical fusion of classifiers. Neuroimage. 2014;90:449-468.

130. Thompson WH, Fransson P. On Stabilizing the Variance of Dynamic Functional Brain Connectivity Time Series. Brain Connect. 2016;6:735-746.

131. Wold S, Esbensen K, Geladi P. Principal component analysis. Chemom Intell Lab Syst. 1987;2:37-52.

132. Alexander-Bloch AF, Shou H, Liu S, Satterthwaite TD, Glahn DC, Shinohara RT, et al. On testing for spatial correspondence between maps of human brain structure and function. Neuroimage. 2018;178:540-551.

133. Benjamini Y, Hochberg Y. Controlling the False Discovery Rate : A Practical and Powerful Approach to Multiple Testing. J R Stat Soc. 1995;57:289-300.

134. Coifman RR, Lafon S. Diffusion maps. Appl Comput Harmon Anal. 2006;21:5-30.

135. von Luxburg U. A tutorial on spectral clustering. Stat Comput. 2007;17:395-416.

136. Park B, Vos de Wael R, Paquola C, Larivière S, Benkarim O, Royer J, et al. Signal diffusion along connectome gradients and inter-hub routing differentially contribute to dynamic human brain function. Neuroimage. 2021;224:117429.

137. Tsamardinos I, Rakhshani A, Lagani V. Performance-Estimation Properties of CrossValidation-Based Protocols with Simultaneous Hyper-Parameter Optimization. Int J Artif Intell Tools. 2015;24:1540023. 
Park et al. | Cross-condition effects of major psychiatric conditions

\section{Supporting Information}

Table S1 | Demographic information of studied participants.

\begin{tabular}{c|c|c|c}
\hline Condition & Number* & Mean (SD; range) age (years) & Sex (male:female) \\
\hline ASD/controls & $1821 / 1823$ & $15.6(6.7 ; 2-64)$ & $2941: 703(19 \%$ female) \\
\hline ADHD/controls & $1815 / 1602$ & $21.1(5.4 ; 4-74)$ & $2244: 1172(34 \%$ female) \\
\hline MDD/controls & $2695 / 3627$ & $40.9(10.9 ; 8-89)$ & $2665: 3657(58 \%$ female) \\
\hline OCD/controls & $2274 / 2013$ & $27.2(8.0 ; 5-65)$ & $2166: 2121(49 \%$ female) \\
\hline BD/controls & $1555 / 3423$ & $35.1(12.0 ; 8-86)$ & $2142: 2836(57 \%$ female) \\
\hline SZ/controls & $2716 / 3272$ & $33.9(10.7 ; 7-87)$ & $3479: 2509(42 \%$ female $)$ \\
\hline
\end{tabular}

Detailed information available in eTable 1 and eTable 2 of Patel et al., 2021. Abbreviations: SD, standard deviation; ASD, autism spectrum disorder; ADHD, attention deficit hyperactivity disorder; MDD, major depressive disorder; OCD, obsessive-compulsive disorder; BD, bipolar disorder; SZ, schizophrenia. 
Park et al. | Cross-condition effects of major psychiatric conditions

\section{A. Other dimensions}
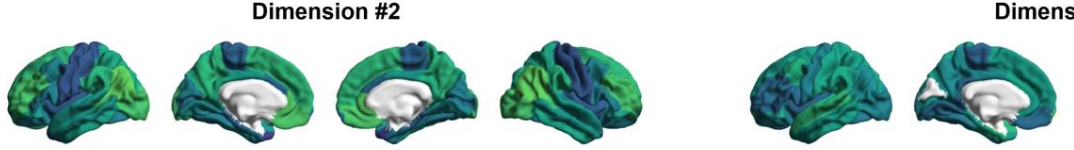

Dimension \#3

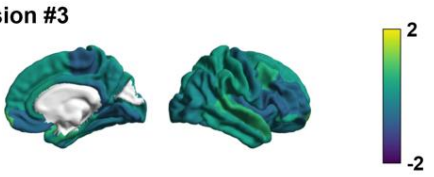

\section{B. Mean effect size}
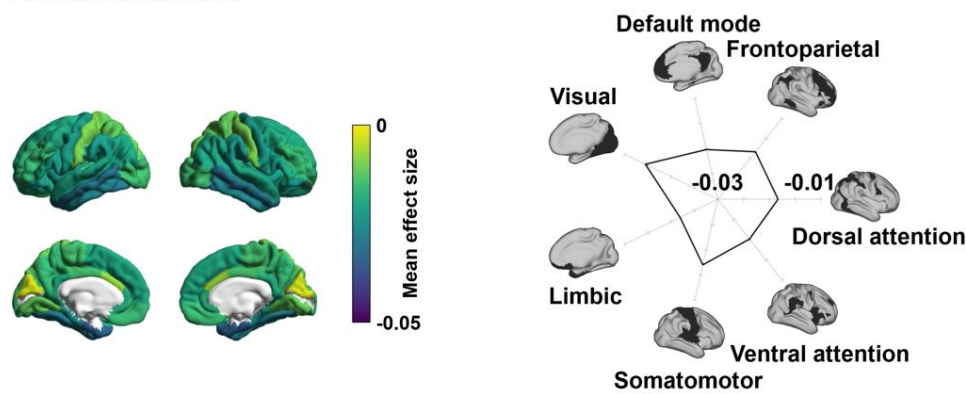

C. Principal dimension sourced from ENIGMA toolbox

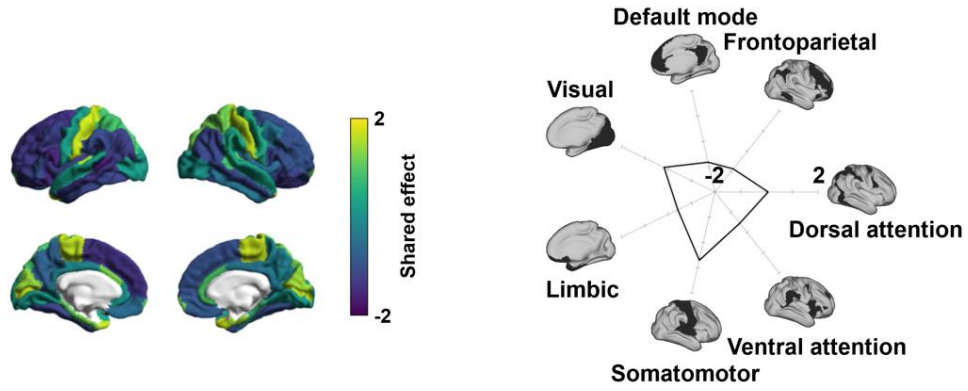

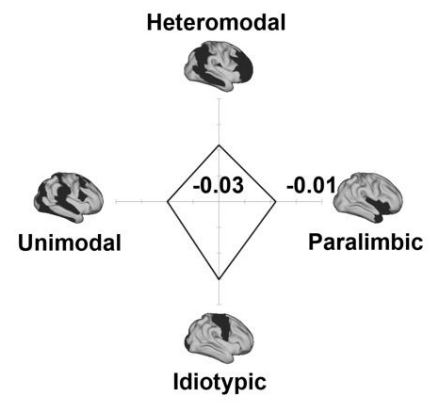

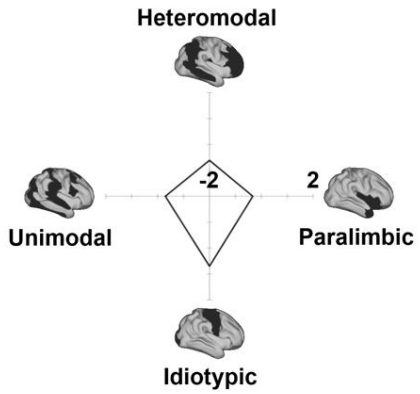

Fig. S1 | Shared disease effect. (A) The second and third dimensions of shared disease effect. (B) Mean effect size of cortical thickness alterations across conditions. (C) Principal dimension based on the effect size maps (Cohen's d) sourced from the ENIGMA toolbox. The effects were stratified according to functional communities and cortical hierarchy levels. 
Park et al. | Cross-condition effects of major psychiatric conditions

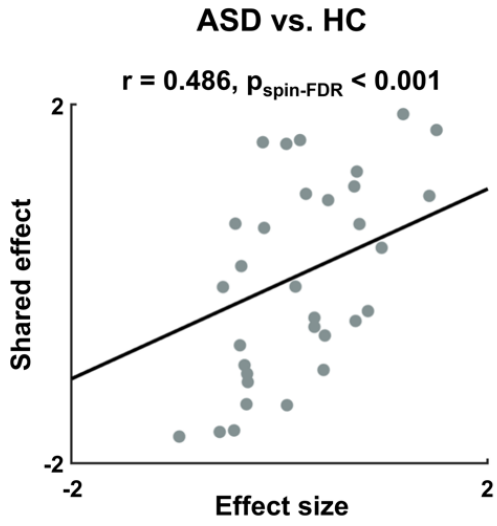

OCD vs. HC

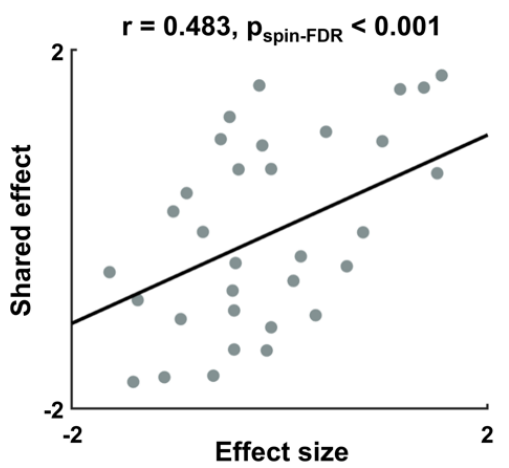

ADHD vs. HC

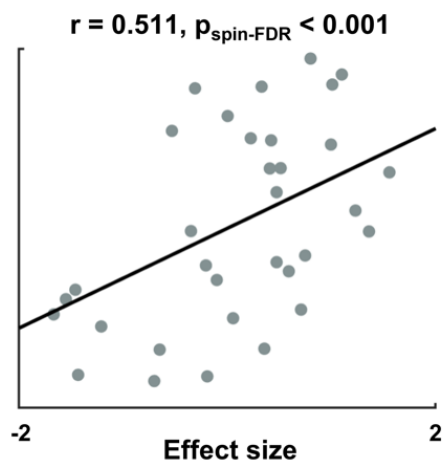

BD vs. HC

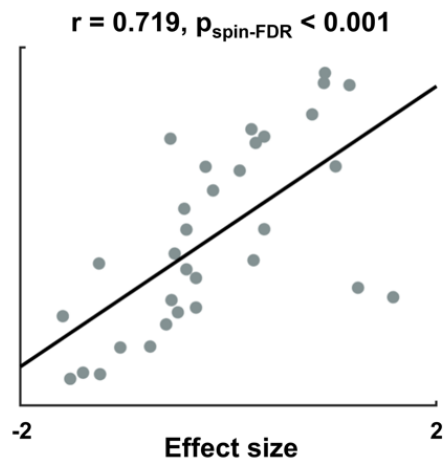

MDD vs. HC

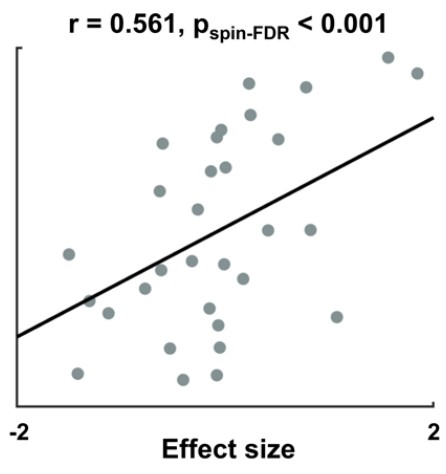

SZ vs. HC

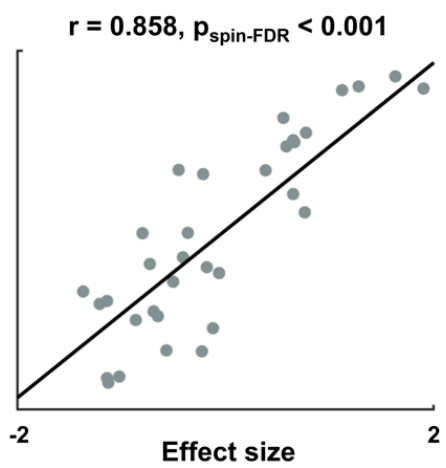

Fig. S2 | Linear correlations between the shared disease effect and cortical thickness alterations of each condition. Abbreviations: ASD, autism spectrum disorder; ADHD, attention deficit hyperactivity disorder; MDD, major depressive disorder; OCD, obsessive-compulsive disorder; BD, bipolar disorder; SZ, schizophrenia; HC, healthy controls. 
Park et al. | Cross-condition effects of major psychiatric conditions
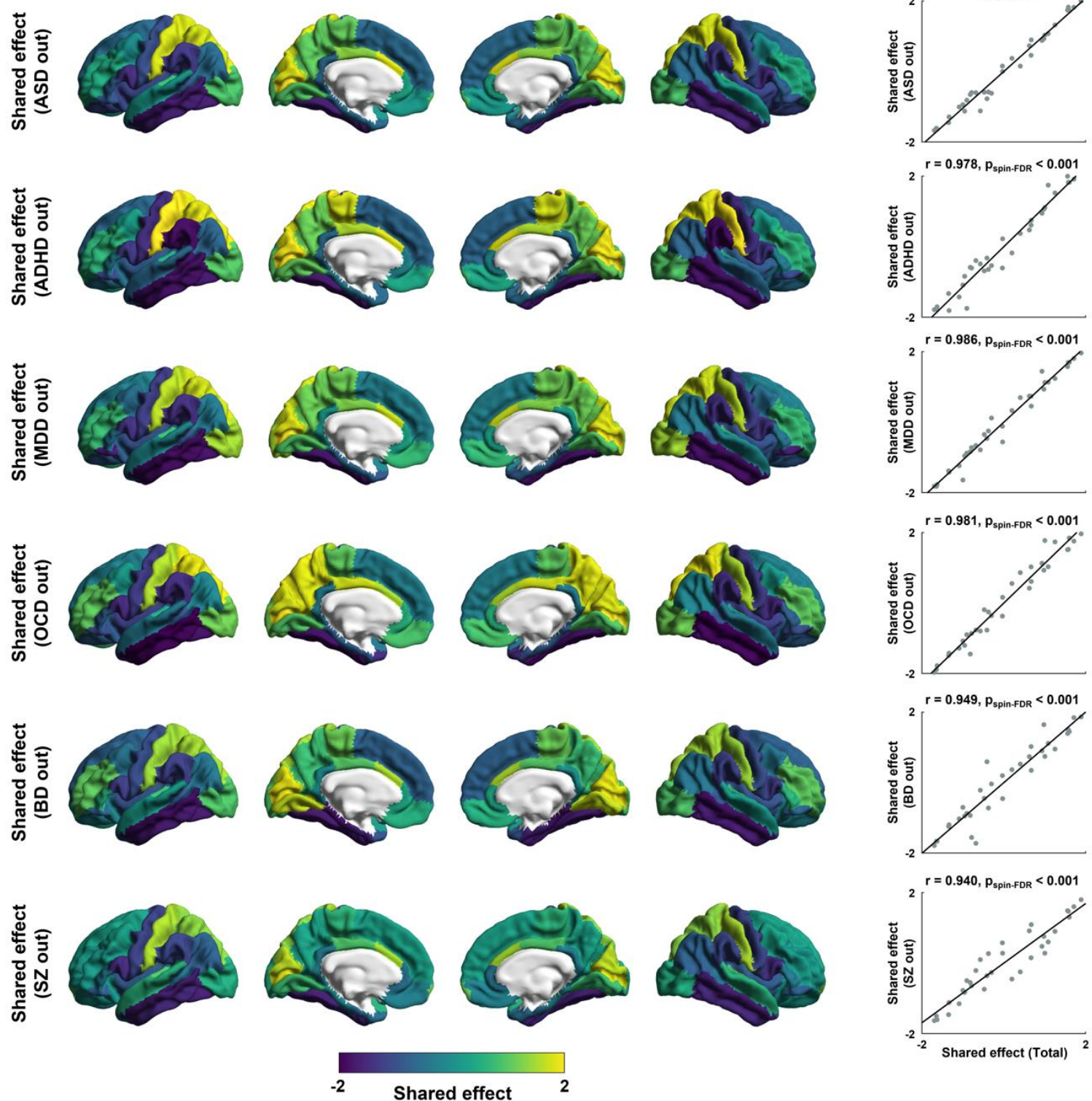

Fig. S3 | Shared disease effects with leave-one-condition-out cross-validation. The shared dimensions estimated based on all conditions without a single condition are reported on brain surfaces. Linear correlations between the shared effect based on all conditions (see Fig. 1B) and that based on five conditions are shown in the scatter plots. Abbreviations: ASD, autism spectrum disorder; ADHD, attention deficit hyperactivity disorder; MDD, major depressive disorder; OCD, obsessivecompulsive disorder; BD, bipolar disorder; SZ, schizophrenia; HC, healthy controls. 
Park et al. | Cross-condition effects of major psychiatric conditions

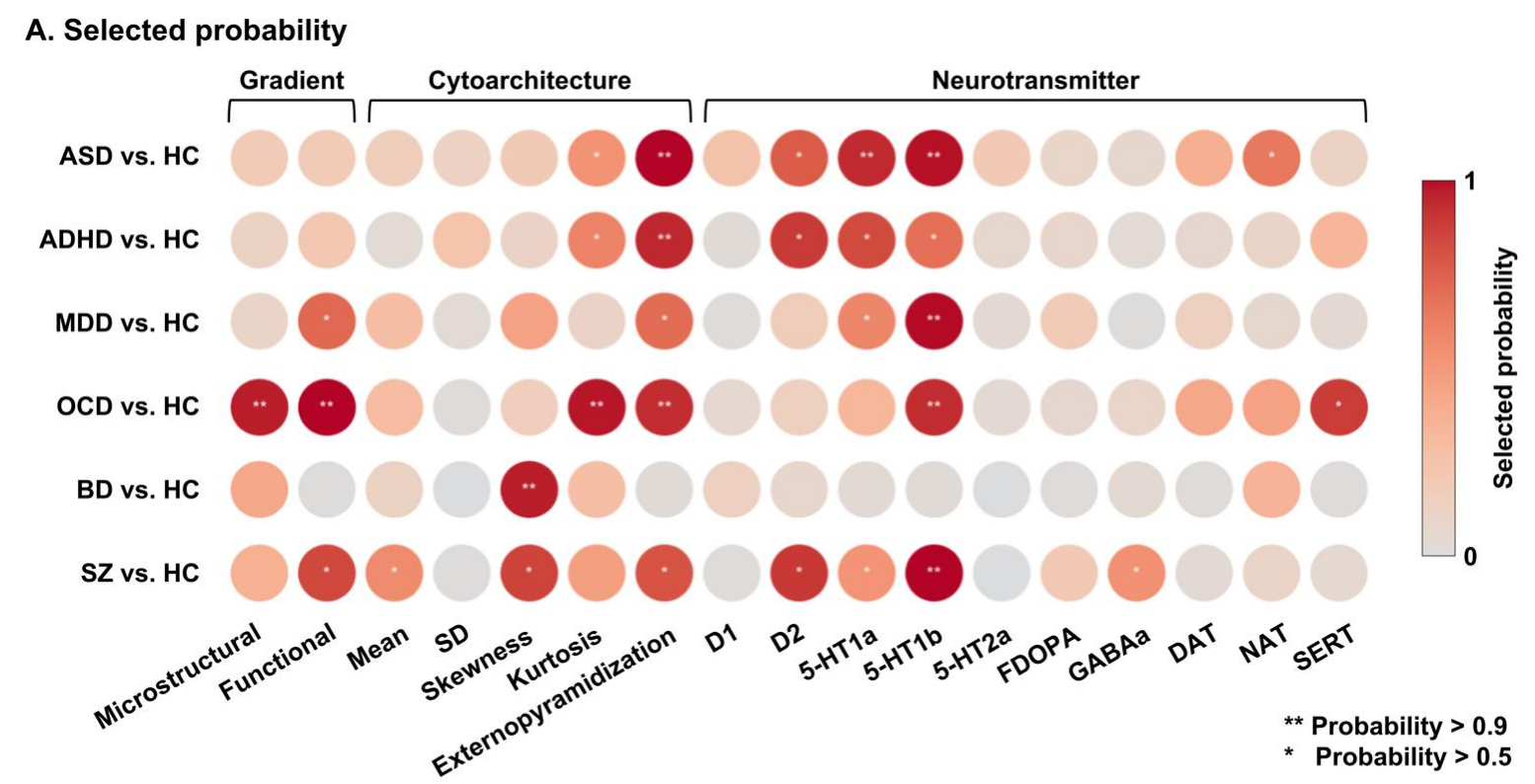

B. Prediction of shared effect and effect size of each condition
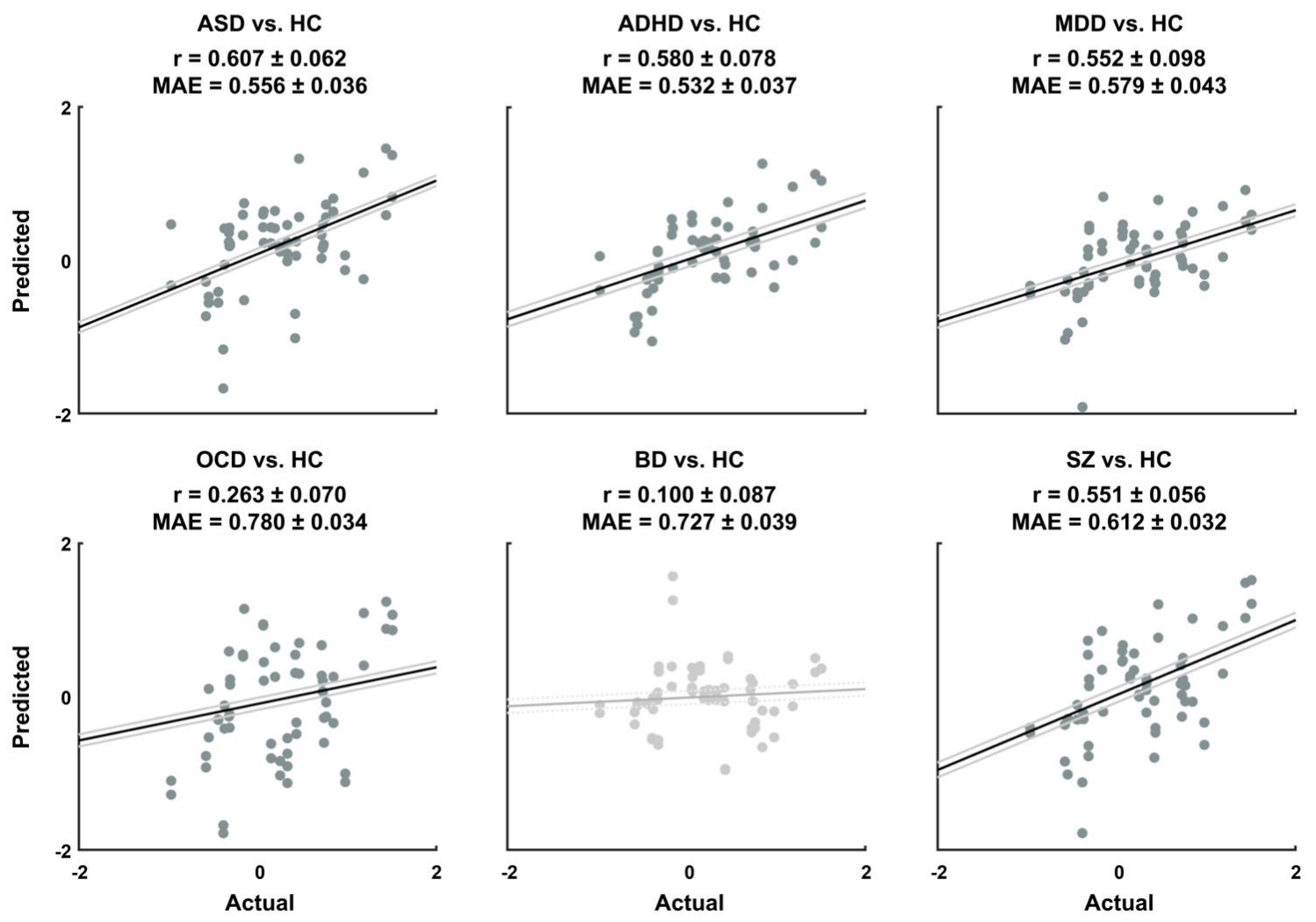

Fig. S4 | Association between the effect size of each psychiatric condition and multiscale features. (A) Probability of the selected features for each psychiatric condition. (B) Linear correlations between actual and predicted values of the effects are shown using scatter plots. For details, see Fig. 4. Abbreviations: SD, standard deviation; FDOPA, 18F fluorodopa; DAT, dopamine transporter; NAT, noradrenaline transporter; SERT, serotonin transporter; MAE, mean absolute error. 